\title{
Resolution of 5-oxo-1-phenylpyrazolidine-3-carboxylic acid and synthesis of novel enantiopure amide derivatives
}

\author{
Roberto Melgar-Fernández, Rodrigo González-Olvera, Jorge Vargas-Caporali, Rosendo \\ Pérez-Isidoro, and Eusebio Juaristi*
}

Departamento de Química, Centro de Investigación y de Estudios Avanzados del Instituto Politécnico Nacional, Apartado Postal 14-740, 07000 México, D. F., México

E-mail: juaristi@relaq.mx

\section{Dedicated to Bruce E. Maryanoff and Cynthia A. Maryanoff, in appreciation for their active role in the promotion of chemical science}

\begin{abstract}
The synthesis is reported of ( \pm )-5-oxo-1-phenylpyrazolidine-3-carboxylic acid, $( \pm)$-3, via nucleophilic addition of phenylhydrazine to dimethyl maleate, followed by cyclization of the resulting hydrazine-diester. The resolution of $( \pm)-3$ was achieved via diastereomeric salts employing $(R)$ - and $(S)$ - $\alpha$-methylbenzylamine as resolving agent. Preferential crystallization of the like $(R, R)$ - and $(S, S)$ - salts allowed the isolation of the desired enantiomerically pure $(R)$ - and $(S)$ - target compounds in up to $87-89 \%$ of the theoretical yield. An X-ray structure of the $(S, S)$ salt allowed secure assignment of its relative configuration, and thus unequivocal assignment of the absolute configuration in the enantiomeric heterocycles.
\end{abstract}

Keywords: Resolution, diastereomeric salts, 5-oxo-1-phenylpyrazolidine-3-carboxylic acid, $\alpha$ methylbenzylamine, chiral amides.

\section{Introduction}

5-Oxo-1-arylpyrazolidines are important heterocycles owing to their potential application as bacterial antibiotics ${ }^{1}$ or as precursors of carbapenems ${ }^{2}$ and diazepinones. ${ }^{3}$ Salient methodologies employed to obtain pyrazolidine derivatives include conjugate addition of hydrazines to $\alpha, \beta$ unsaturated imides, ${ }^{4}$ insertion of diazene derivatives into the cyclopropane ring $^{5}$ and conjugate addition-rearrangement of hydrazines to $\alpha, \beta$-unsaturated sugar $\delta$-lactones. ${ }^{1}$ However, these methods present some drawbacks, such as low chemoselectivity and enantioselectivity.

In 2006, we reported the preparation of $(R)$ - and (S)-5-oxo-1-phenylpyrazolidine-3carboxylic acid, $(R)$ - and $(S)-3$ via resolution with $\alpha$-methylbenzylamine. ${ }^{6}$ Soon thereafter, Tzeng 
and coworkers independently described the resolution of ( \pm -3 with L-amino-acid methyl esters. ${ }^{7}$ We now provide full details of the efficient resolution of 5-oxo-1-phenylpyrazolidine-3carboxylic acid with $\alpha$-methylbenzylamine as resolving agent, ${ }^{8}$ as well as the preparation of several enantiopure amide derivatives.

\section{Results and Discussion}

The racemic dimethyl 2-(2-phenylhydrazinyl)succinate, $( \pm)-2$, was prepared from dimethyl maleate 1 by means of nucleophilic addition of phenylhydrazine in methanol at room temperature. Compound $( \pm)-2$ was obtained with $89 \%$ yield (Scheme 1$){ }^{9}$

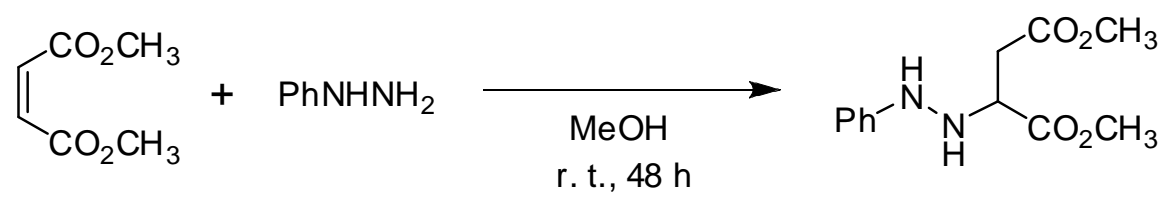

1

$89 \%$

$( \pm)-2$

\section{Scheme 1}

The hydrazine-diester $( \pm)-2$ was treated with sodium methoxide to give the heterocyclic ester $( \pm)-5$ in $44 \%$ isolated yield (Scheme 2$).{ }^{9}$ The ${ }^{1} \mathrm{H}$ - NMR spectrum for heterocycle $( \pm$ ) 5 showed a doublet signal at $5.27 \mathrm{ppm}$, which was assigned to the exchangeable $\mathrm{NH}$ proton and a doublet of doublet of doublets signal in $4.36 \mathrm{ppm}$ for the tertiary $\mathrm{CH}$ proton. The observed coupling between the proton of the methine group and a $\mathrm{NH}$ proton led to the conclusion that the phenyl substituent must be located at the $\alpha$-nitrogen relative to the endocyclic carbonyl group.

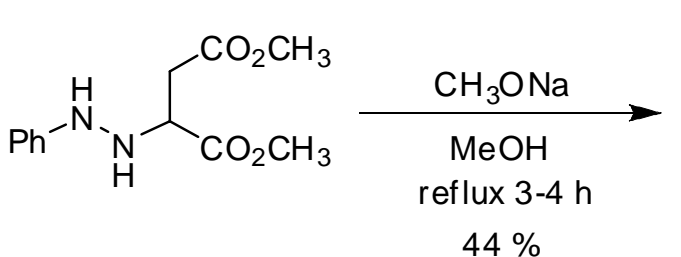

$( \pm)-2$

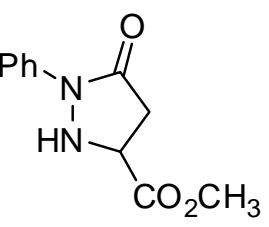

$( \pm)-5$

\section{Scheme 2}

An X-ray structure of $( \pm)-5$, confirmed the conclusions reached from the analysis of the ${ }^{1} \mathrm{H}-$ NMR spectra; that is, the phenyl substituent is bound to the $\alpha$-nitrogen (Figure 1). 


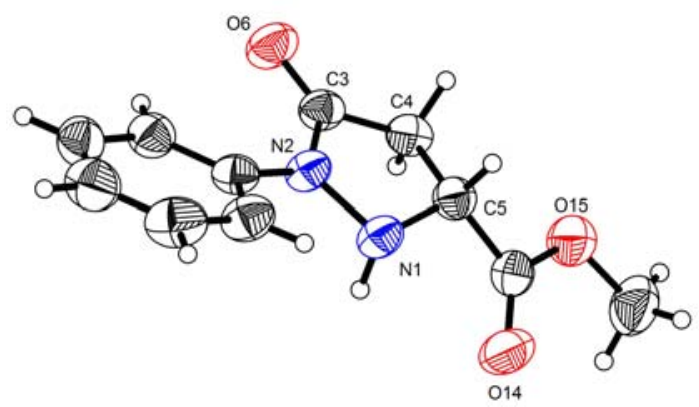

Figure 1. X-ray crystallographic structure of $( \pm)-5$ showing the phenyl ring bound to the $\alpha$ nitrogen. $^{14}$

The preparation of ( \pm )-5-oxo-1-phenylpyrazolidine-3-carboxylic acid, ( \pm )-3, by direct cyclization of the hydrazine-diester $( \pm)-2$ was achieved with potassium carbonate in $\mathrm{MeOH}-\mathrm{H}_{2} \mathrm{O}$, by heating the reaction mixture under microwave irradiation during 15 minutes, followed by treatment with concentrated $\mathrm{HCl}^{9}{ }^{9}$ The desired product was obtained in $83 \%$ isolated yield (Scheme 3).

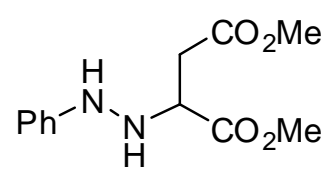

$( \pm)-2$

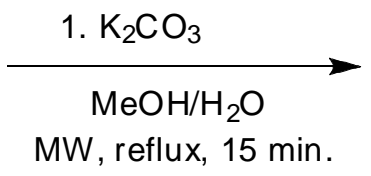

2. $\mathrm{HCl}$

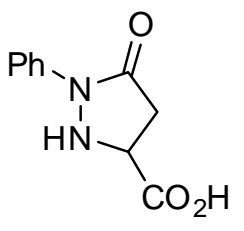

$( \pm)-3$

$83 \%$

\section{Scheme 3.}

Enantiomerically pure $\alpha$-methylbenzylamine has been employed widely as a resolving agent; ${ }^{8,10}$ thus, the next step consisted in the resolution of $( \pm)$-3 via diastereomeric salt formation with this chiral amine. The racemic acid $( \pm)$-3 was dissolved in hot ethyl acetate-isopropanol $(70: 30 \mathrm{v} / \mathrm{v})$ before the addition of half equivalent of $(R)$ - or $(S)$ - $\alpha$-methylbenzylamine. One diastereomeric salt precipitated from the solution whereas the other one remained in solution. Each diastereomeric salt was obtained in $85 \%$ of the theoretical yield (Scheme 4).

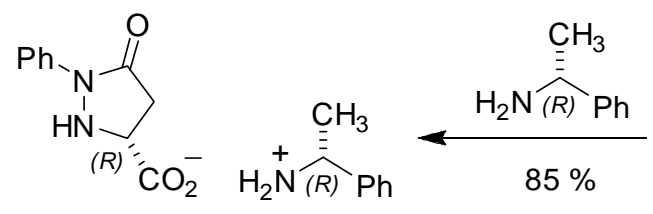

$(R, R)-4$

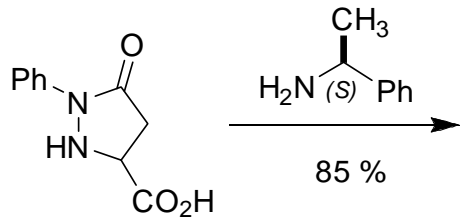

$( \pm)-3$

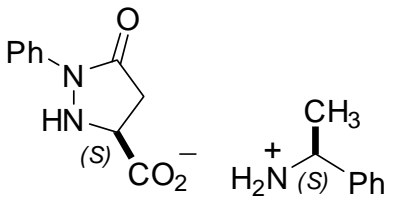

$(S, S)-4$

Scheme 4. Resolution of $( \pm)$-3 with $(R)$ - and $(S)$ - $\alpha$-methylbenzylamines. 
An X-ray crystallographic structure of the insoluble diastereomeric salt formed with $(S)-\alpha-$ methylbenzylamine allowed for identification of the like ${ }^{11}$ relative configuration, and therefore to the assignment of the $(S)$ - absolute configuration of the stereogenic center of the heterocycle. With this information we determined that precipitation of the like salt is favored (Figure 2).

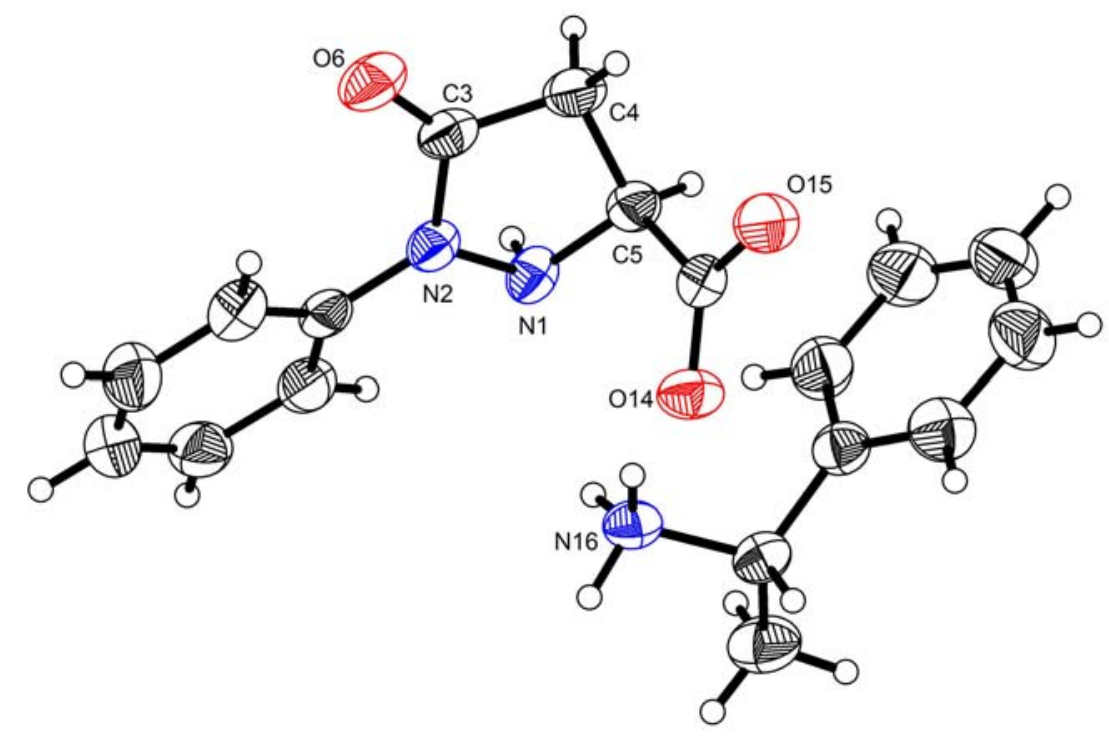

Figure 2. X-ray crystallographic structure of $(S, S)$-4 showing the like $(S, S)$ relative configuration of the stereogenic centers. ${ }^{14}$

The like $(R, R)-\mathbf{4}$ and $(S, S)-\mathbf{4}$ salts were dissolved in aqueous medium and treated with $\mathrm{HCl}$ to recover both enantiomers of $\mathbf{3}$ in free form by filtering the precipitate. Afterward, acids $(R)$ - and $(S)$-3 were transformed into their corresponding methyl esters through a Fischer's esterification (Scheme 5). The methyl esters $(R)$ - and $(S)$-5 were analyzed by chiral HPLC and both showed an enantiomeric excess $>99 \%$ (see the Experimental Section). Subsequently, protection of the $\beta$ nitrogen in enantiomerically pure methyl esters $(R)$ - and $(S)$-5 was carried out using 4 equivalents of (Boc) $)_{2} \mathrm{O}$, obtaining $(R)$ - and $(S)-6$ in good yield. Saponification of $N$-Boc esters 6 was achieved with lithium hydroxide monohydrate in THF. The expected acids $(R)-$ and $(S)-7$ were obtained in 83 and $90 \%$ yields, respectively (Scheme 6). 


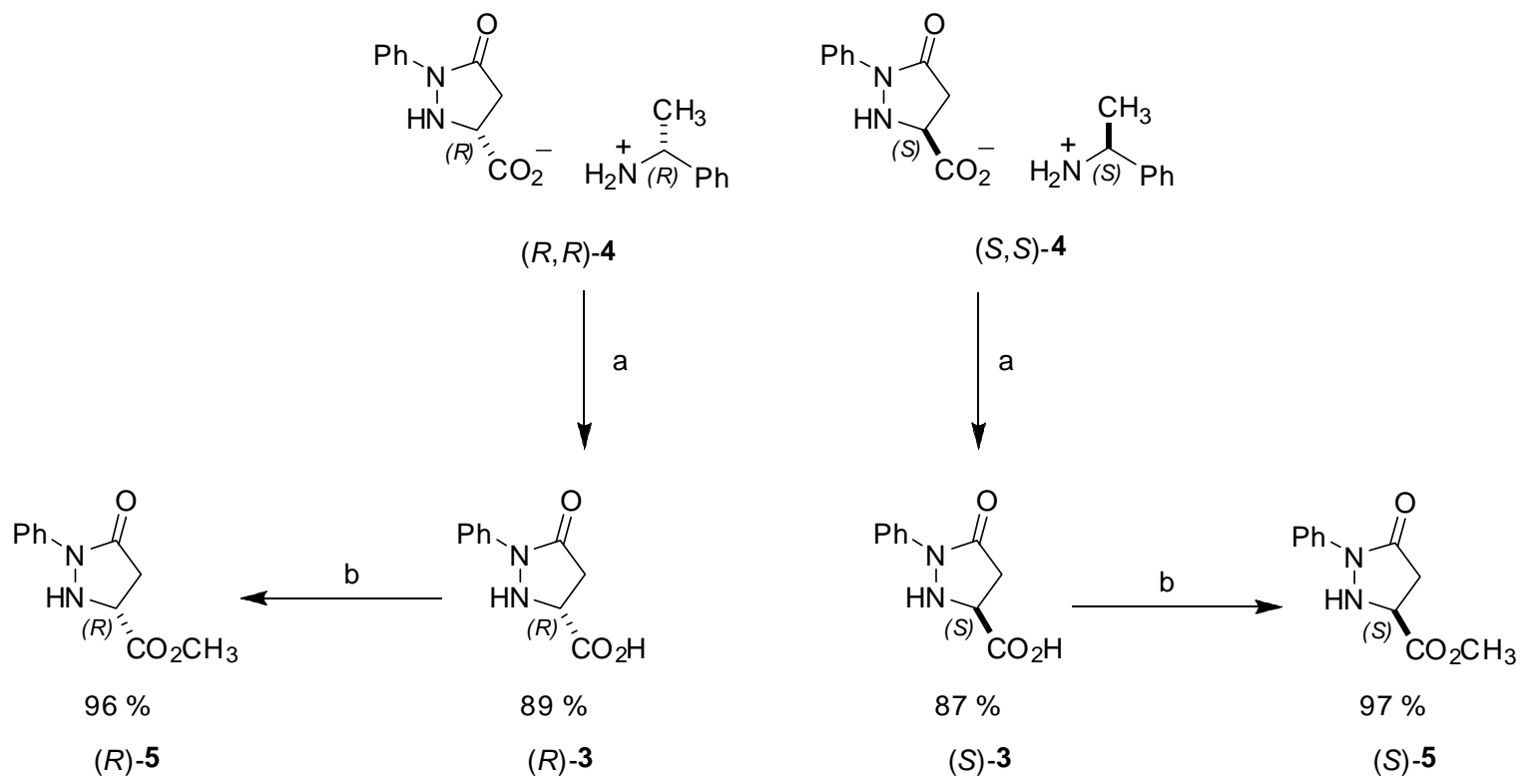

Scheme 5. Reaction conditions: (a) $\mathrm{HCl}$; (b) $\mathrm{H}_{2} \mathrm{SO}_{4}$, methanol, $\mathrm{MW}$, reflux, 10 minutes.

The coupling reaction was carried out through the formation of a mixed anhydride with pivaloyl chloride before the addition of the corresponding amine (aniline, benzylamine, $(S)-\alpha-$ methylbenzylamine, or (S)-2-amino-1-butanol) and heating to reflux for 30 minutes. The protected amides 8-11 were obtained with yields from 69 to $88 \%$ (Table 1). An X-ray structure was obtained for compound $(R, S)$-11. Salient observations are the anti-parallel orientation of the carbonyl groups and the lack of hydrogen bonding between the hydroxyl group and the N-H amide function (Figure 3).

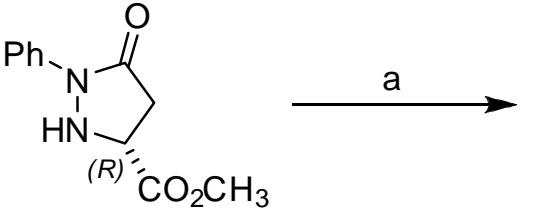

$(R)-5$

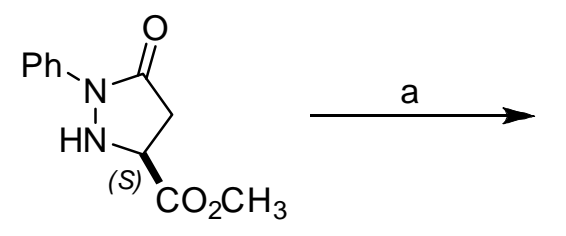

(S) -5<smiles>COC(=O)[C@H]1CC(=O)N(c2ccccc2)N1C(=O)OC(C)(C)C</smiles>

$87 \%$

$(R)-6$

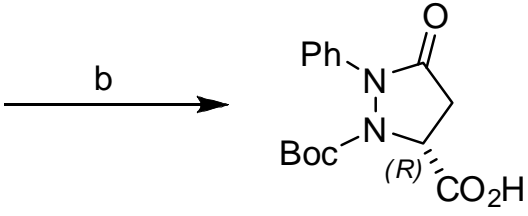

$83 \%$

$(R)-7$<smiles>COC(=O)[C@H]1CC(=O)N(c2ccccc2)N1C(=O)OC(C)(C)C</smiles>

$89 \%$

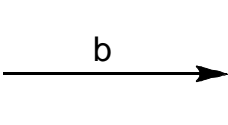

(S)-6<smiles>CC(C)(C)OC(=O)N1C(=O)CC(C(=O)O)N1c1ccccc1</smiles>

$90 \%$

Scheme 6. Reaction conditions: (a) $\mathrm{Boc}_{2} \mathrm{O}, \mathrm{DMAP}, \mathrm{Et}_{3} \mathrm{~N}, \mathrm{CH}_{2} \mathrm{Cl}_{2}, \mathrm{RT}, 48 \mathrm{~h}$; (b) $\mathrm{LiOH} \cdot \mathrm{H}_{2} \mathrm{O}$, THF, RT, 44 h. 
Table 1. Coupling reaction of $(R)-7$ and $(S)-7$ with several amines to afford enantiopure amides (R)-8-11 and (S)-8-11.
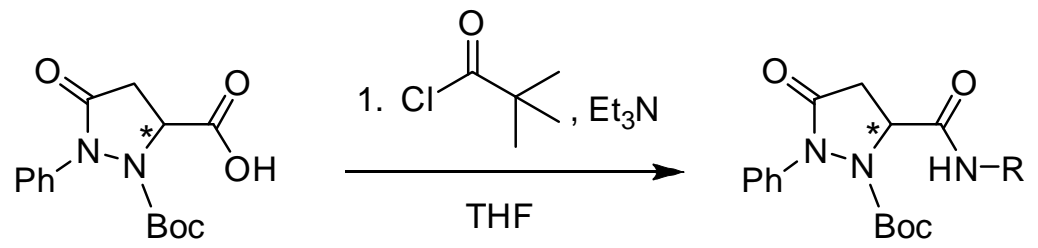

(R)- or (S)-7

2. $\mathrm{H}_{2} \mathrm{~N}-\mathrm{R}$

(R)- or (S)-8-11

Isolated Yield




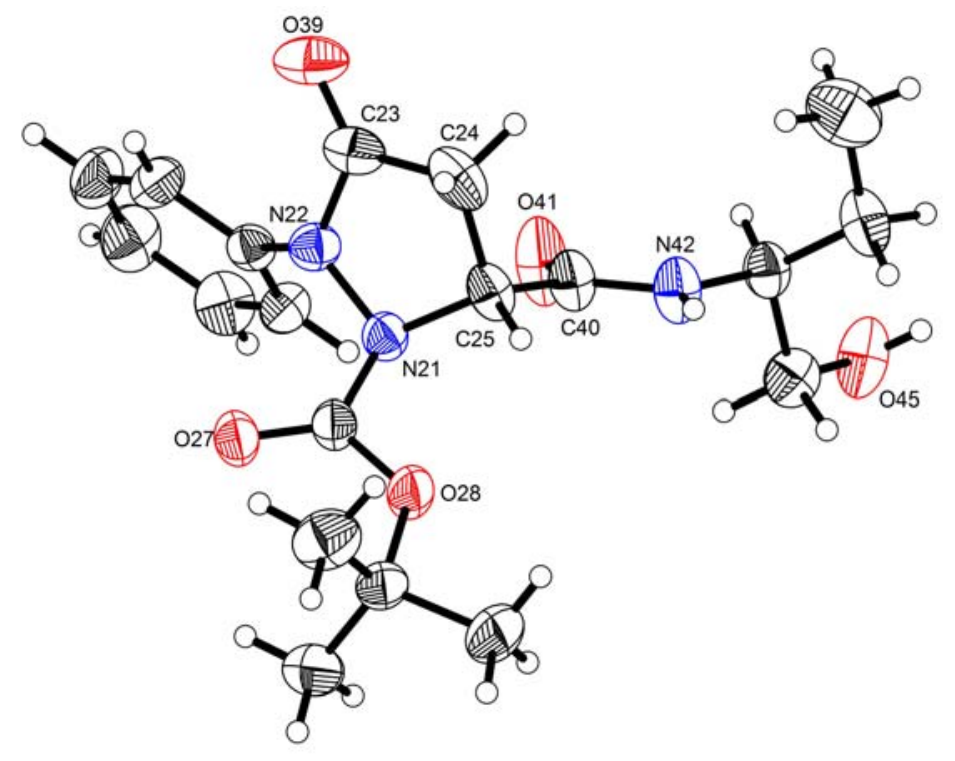

Figure 3. X-ray crystallographic structure of $(R, S)-\mathbf{1 1} .^{14}$

Finally, the $N$-Boc protecting group was removed with trifluoroacetic acid to obtain the compounds of interest $(R)-\mathbf{1 2 - 1 5}$ and $(S)-\mathbf{1 2 - 1 5}$ in good yields (Table 2). X-ray crystallographic structures were obtained for compounds $(S)-\mathbf{1 2}$ and $(S, S)$-15. It can be appreciated in both structures that the carbonyl groups are oriented in parallel (Figure 4).
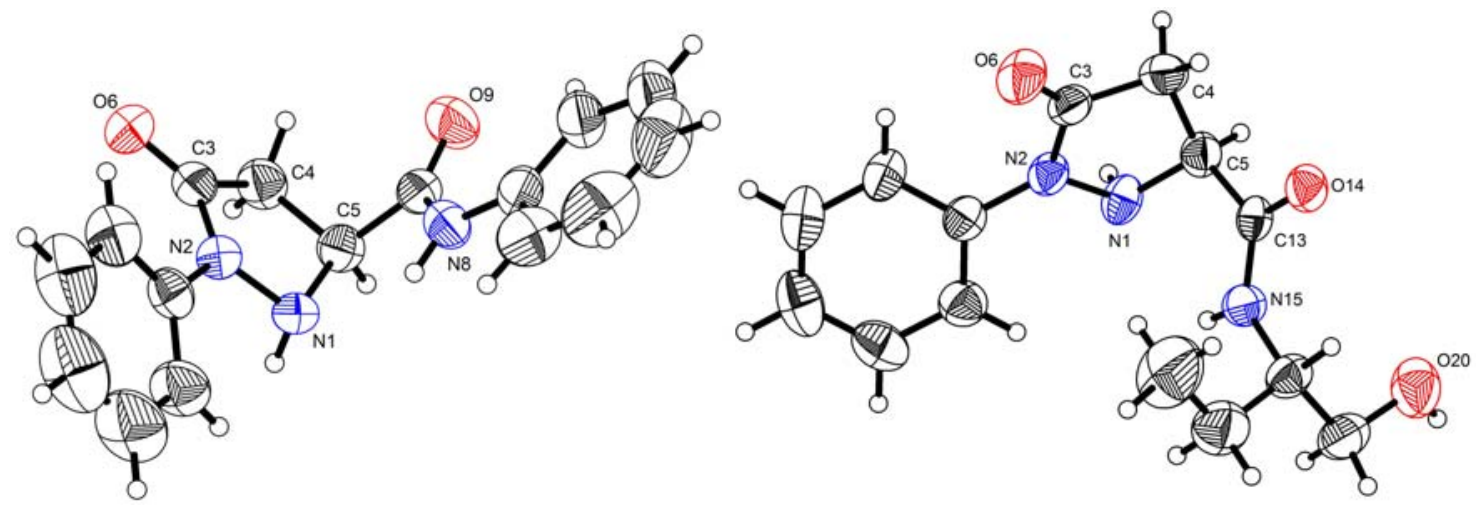

Figure 4. X-ray crystallographic structures of (S)-12 (left) and (S,S)-15 (right). ${ }^{14}$ 
Table 2. Removal of the $N$-Boc group with trifluoroacetic acid.<smiles>[R]NC(=O)C1=NN(c2ccccc2)C(=O)C1</smiles>

$(R)$ or $(S)$

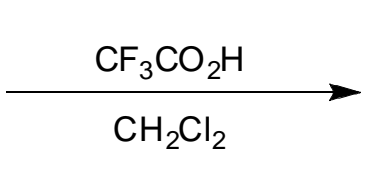

Product

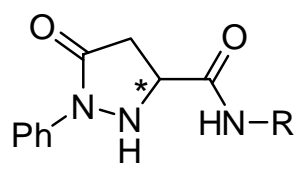

$(R)$ or $(S)$

Isolated Yield
$(\%)$

\section{Conclusions}

An efficient method for the preparation of racemic 5-oxo-1-phenylpyrazolidine-3-carboxylic acid and its subsequent resolution via separation of diastereomeric salts, in three steps with $85 \%$ overall yield, has been developed. These chiral acids and several novel amide derivatives reported herein are presently being evaluated as building blocks, ligands, and/or organo-catalysts in asymmetric synthesis. 


\section{Experimental Section}

General. Optical rotations were measured in a Perkin-Elmer 241 polarimeter. Melting points were measured on a Melt-Temp 'Electrothermal' apparatus and are uncorrected. NMR spectra were recorded in Bruker Advance $300(300 \mathrm{MHz})$ and JOEL Eclipse+400 (400 MHz) spectrometers. IR spectra were recorded on a Perkin-Elmer FTIR spectrum-GX apparatus. Mass spectra were registered on a Hewlett Packard 5989-AMS-ENGINE, Thermo Electron TraceDSQ spectrometer, at $20 \mathrm{eV}$. HRMS were recorded on JEOL JMS-SX 102a and Agilent-MSDTOF1069A spectrometers. Elemental analyses were obtained using a Thermo-Finnigan CHNS/O 1112 apparatus. The structural X-ray crystallographic data were obtained on an Enraf-Nonius Kappa CCD diffractometer. Microwave irradiation was achieved in a single-mode Discover System reactor from CEM Corporation. HPLC analyses were carried out with a Waters $600 \mathrm{E}$ equipment fitted with a UV/Visible Waters 2487 detector at $230 \mathrm{~nm}$ and Chiralpack AD (Daicel Chemical Ind., LTD, 0.46 x $25 \mathrm{~cm}$ ) column, employing hexane-i-PrOH (90:10) as mobile phase, and $1 \mathrm{~mL} / \mathrm{min}$ flow.

Dimethyl maleate 1. In a $500 \mathrm{~mL}$ three-necked round-bottomed flask equipped with a magnetic stirrer and a reflux condenser, $35 \mathrm{~g}(301.7 \mathrm{mmol})$ of maleic acid were placed and dissolved with $200 \mathrm{~mL}$ of methanol before the addition of $3.5 \mathrm{~g}(18.4 \mathrm{mmol}, 10 \mathrm{w} / \mathrm{w} \%)$ of $p$-toluenesulfonic acid and the flask was heated to reflux for $11 \mathrm{~h}$. After this time, the solvent was concentrated under vacuum and the crude reaction mixture was treated with a saturated solution of $\mathrm{NaHCO}_{3}$ $(100 \mathrm{~mL})$ and extracted with ethyl acetate $(3 \times 30 \mathrm{~mL})$. The organic layer was dried with anhydrous $\mathrm{Na}_{2} \mathrm{SO}_{4}$ and concentrated under vacuum to provide 1 (36.2 g, $84 \%$ yield) as a colorless oil. ${ }^{1} \mathrm{H}-\mathrm{NMR}\left(\mathrm{CDCl}_{3}, 270 \mathrm{MHz}\right): \delta=6.22(\mathrm{~s}, 2 \mathrm{H}, \mathrm{HC}=\mathrm{CH}), 3.77\left(\mathrm{~s}, 6 \mathrm{H}, \mathrm{OCH}_{3}\right) .{ }^{13} \mathrm{C}-$ $\operatorname{NMR}\left(\mathrm{CDCl}_{3}, 67.9 \mathrm{MHz}\right): \delta=165.7(\mathrm{C}=\mathrm{O}), 129.8(\mathrm{C}=\mathrm{C}), 52.2\left(\mathrm{OCH}_{3}\right)$.

( \pm -Dimethyl 2-(2-phenylhydrazinyl)succinate, $( \pm)-2$. In a $250 \mathrm{~mL}$ round-bottomed flask equipped with a magnetic stirrer and an addition funnel, $8.4 \mathrm{~g}(58.33 \mathrm{mmol})$ of dimethyl maleate 1 were placed and dissolved in $80 \mathrm{~mL}$ of methanol under nitrogen atmosphere. Subsequently, $4.8 \mathrm{~mL}$ ( $46.61 \mathrm{mmol}, 5.25 \mathrm{~g}$ ) of phenylhydrazine was added slowly and the reaction mixture was stirred during $48 \mathrm{~h}$ at room temperature. After this time, approximately one third of the solvent was evaporated under vacuum and the flask was cooled to $0{ }^{\circ} \mathrm{C}$, observing a precipitate which corresponded to dimethyl fumarate $(1.15 \mathrm{~g}),{ }^{12}$ which was filtered off, and washed twice with cold methanol $(15 \mathrm{~mL})$. The filtrate was evaporated under vacuum to provide a solid, which was suspended in hexane $(15 \mathrm{~mL})$ and filtered affording $10.5 \mathrm{~g}$ of $( \pm)-2(89 \%$ yield $)$ as a yellow solid, $\mathrm{mp} 44-46{ }^{\circ} \mathrm{C} .{ }^{1} \mathrm{H}-\mathrm{NMR}\left(\mathrm{CDCl}_{3}, 400 \mathrm{MHz}\right): \delta=2.82\left(\mathrm{dd}, J=6.9,16.4 \mathrm{~Hz}, 1 \mathrm{H}, \mathrm{CH}_{2}\right), 2.87(\mathrm{dd}, J$ $\left.=5.1,16.3 \mathrm{~Hz}, 1 \mathrm{H}, \mathrm{CH}_{2}\right), 3.71\left(\mathrm{~s}, 3 \mathrm{H}, \mathrm{OCH}_{3}\right), 3.78\left(\mathrm{~s}, 3 \mathrm{H}, \mathrm{OCH}_{3}\right), 3.95(\mathrm{dd}, J=5.3,6.6 \mathrm{~Hz}, 1 \mathrm{H}$, $\mathrm{CH}), 4.41(\mathrm{br}, 1 \mathrm{H}, \mathrm{NH}), 5.74(\mathrm{br}, 1 \mathrm{H}, \mathrm{NH}), 6.76-6.82(\mathrm{~m}, 1 \mathrm{H}, \mathrm{ArCH}), 6.84-6.91(\mathrm{~m}, 2 \mathrm{H}, \mathrm{ArCH})$, 7.16-7.23 (m, 2H, $\mathrm{ArCH}) .{ }^{13} \mathrm{C}-\mathrm{NMR}\left(\mathrm{CDCl}_{3}, 100.5 \mathrm{MHz}\right): \delta=35.6\left(\mathrm{CH}_{2}\right), 52.0\left(\mathrm{OCH}_{3}\right), 52.5$ $\left(\mathrm{OCH}_{3}\right), 59.2(\mathrm{CH}), 113.1(2 \mathrm{xArCH}), 119.4(\mathrm{ArCH}), 129.1(2 \mathrm{xArCH}), 149.0\left(C_{\mathrm{ipso}}\right), 171.5$ $(C=\mathrm{O}), 173.7(C=\mathrm{O})$. MS (IE) m/z (\%): $252\left(\mathrm{M}^{+}, 88\right), 193$ (46), 161 (37), 133 (15), 119 (70); 107 
(100), 92 (99), 77 (67), 65 (63). IR $v_{\max }(\mathrm{KBr}) \mathrm{cm}^{-1}:$ 3324, 2954, 1739, 1630, 1603, 1437, 1220, 996, 754. Anal. Calcd. for $\mathrm{C}_{12} \mathrm{H}_{16} \mathrm{~N}_{2} \mathrm{O}_{4}$ (252.27): C 57.13, H 6.39, N 11.10; Found: C 57.42, H $6.31, \mathrm{~N} 11.18 \%$.

( \pm )-5-Oxo-1-phenylpyrazolidine-3-carboxylic acid ( \pm-3$)$. In a $100 \mathrm{~mL}$ round-bottomed flask equipped with a magnetic stirrer and a reflux condenser, $3.12 \mathrm{~g}(12.3 \mathrm{mmol})$ of $( \pm)-2$ was placed and dissolved in $30 \mathrm{~mL}$ of methanol before the addition of $1.7 \mathrm{~g}(12.3 \mathrm{mmol})$ of $\mathrm{K}_{2} \mathrm{CO}_{3}$ dissolved in $18 \mathrm{~mL}$ of water. The reaction mixture was set in the microwave equipment and maintained at reflux for 15 min with 75 Watts of power. The methanol solvent was evaporated and $50 \mathrm{~mL}$ of water was added before addition of concentrated $\mathrm{HCl}$ to acidify until $\mathrm{pH} 2.0$, at which point the desired product precipitated. The solid was filtered off, washed with water and dried under vacuum at $40{ }^{\circ} \mathrm{C}$ during $48 \mathrm{~h}$. It was then recrystallized twice from hot methanol and dried under vacuum to give the product $( \pm)-3(2.1 \mathrm{~g}, 83 \%$ yield $)$ as a white powder, mp $210-212{ }^{\circ} \mathrm{C}$ [Lit. ${ }^{13}$ mp 197-199 ${ }^{\circ} \mathrm{C}$ ]. ${ }^{1} \mathrm{H}-\mathrm{NMR}$ (DMSO- $\left.d_{6}, 400 \mathrm{MHz}\right): \delta=2.76\left(\mathrm{dd}, J=5.9,16.5 \mathrm{~Hz}, 1 \mathrm{H}, \mathrm{CH}_{2}\right.$ ), $2.98\left(\mathrm{dd}, J=8.6,16.5 \mathrm{~Hz}, 1 \mathrm{H}, \mathrm{CH}_{2}\right), 4.24\left(\mathrm{dd}, J=5.9,8.1 \mathrm{~Hz}, 1 \mathrm{H}, \mathrm{CH}_{2}\right), 6.55(\mathrm{br}, 1 \mathrm{H}, \mathrm{NH})$, 7.05-7.11 (m, 1H, $\mathrm{ArCH}), 7.31-7.39(\mathrm{~m}, 2 \mathrm{H}, \mathrm{ArCH}), 7.75-7.82(\mathrm{~m}, 2 \mathrm{H}, \mathrm{ArCH}), 13.0(\mathrm{br}, 1 \mathrm{H}$, $\left.\mathrm{CO}_{2} \mathrm{H}\right) .{ }^{13} \mathrm{C}$ - NMR (DMSO- $\left.d_{6}, 100.5 \mathrm{MHz}\right): \delta=37.8\left(\mathrm{CH}_{2}\right), 55.4(\mathrm{CH}), 118.4(2 \mathrm{xArCH}), 124.1$ $(\mathrm{ArCH}), 129.0(2 \mathrm{xArCH}), 139.5\left(C_{\mathrm{ipso}}\right), 170.6(\mathrm{NC}=\mathrm{O}), 172.9\left(\mathrm{CO}_{2} \mathrm{H}\right) . \mathrm{MS}(\mathrm{IE}) \mathrm{m} / \mathrm{z}(\%): 206$ $\left(\mathrm{M}^{+}, 100\right), 161$ (55), 118 (13), 107 (35), 91 (17), 77 (20), 55 (5).

(R)-1-Phenylethylamonium $(R)$-5-oxo-1-phenylpyrazolidine-3-carboxylate, $(R, R-4)$. In a $1 \mathrm{~L}$ three-necked round-bottomed flask equipped with a magnetic stirrer, an addition funnel and a reflux condenser, $10.5 \mathrm{~g}(50.9 \mathrm{mmol})$ of $( \pm)-3$ was dissolved in $570 \mathrm{~mL}$ of EtOAc-i-PrOH $(70: 30, v / v)$ and the resulting mixture was heated until the solution became homogeneous. Subsequently, $3.1 \mathrm{~g}(25.45 \mathrm{mmol}, 3.24 \mathrm{~mL})$ of $(R)$ - $\alpha$-methylbenzylamine was added and the reaction mixture was heated to reflux for $15 \mathrm{~min}$, at which point the expected like salt precipitates (see main text). The heating was stopped and the mixture stirred until it reached room temperature. The reaction mixture was then cooled in an ice-bath for $30 \mathrm{~min}$ and the precipitate filtered, washed with $30 \mathrm{~mL}$ of EtOAc, and dried under vacuum at $40{ }^{\circ} \mathrm{C}$ during $24 \mathrm{~h}$ to obtain $7.0 \mathrm{~g}$ of $(R, R)-4$ (85\% yield) as a white solid, mp $190-192{ }^{\circ} \mathrm{C},[\alpha]_{\mathrm{D}}{ }^{20}=+63.0(\mathrm{c} 2$, MeOH). ${ }^{1} \mathrm{H}-\mathrm{NMR}$ (DMSO- $\left.d_{6}, 400 \mathrm{MHz}\right): \delta=1.48\left(\mathrm{~d}, J=6.8 \mathrm{~Hz}, 3 \mathrm{H}, \mathrm{CH}_{3}\right), 2.70(\mathrm{dd}, J=9.4$, $\left.16.6 \mathrm{~Hz}, 1 \mathrm{H}, \mathrm{CH}_{2}\right), 2.75\left(\mathrm{dd}, J=8.8,16.7 \mathrm{~Hz}, 1 \mathrm{H}, \mathrm{CH}_{2}\right), 3.82\left(\mathrm{dd}, J=8.8,9.2 \mathrm{~Hz}, 1 \mathrm{H},{ }^{*} \mathrm{CH}\right)$, $4.33\left(\mathrm{q}, J=6.7 \mathrm{~Hz}, 1 \mathrm{H},{ }^{*} \mathrm{CH}\right), 7.04-7.08(\mathrm{~m}, 1 \mathrm{H}, \mathrm{ArCH}), 7.29-7.39(\mathrm{~m}, 5 \mathrm{H}, \mathrm{ArCH}), 7.47-7.51$ (m, $2 \mathrm{H}, \mathrm{ArCH}), 7.78-7.81(\mathrm{~m}, 2 \mathrm{H}, \mathrm{ArCH}) .{ }^{13} \mathrm{C}-\mathrm{NMR}$ (DMSO-d $\left.6,100.5 \mathrm{MHz}\right): \delta=21.9\left(\mathrm{CH}_{3}\right)$, $39.1\left(\mathrm{CH}_{2}\right), 50.4\left({ }^{*} \mathrm{CH}\right), 57.4\left({ }^{*} \mathrm{CH}\right), 118.2(2 \times \mathrm{ArCH}), 123.8(\mathrm{ArCH}), 127.2(2 \mathrm{xArCH}), 128.6$ $(\mathrm{ArCH}), 129.0(2 \mathrm{xArCH}), 129.1(2 \times \mathrm{x} C \mathrm{H}), 139.7\left(C_{\mathrm{ipso}}\right), 141.2\left(C_{\mathrm{ipso}}\right), 170.9(\mathrm{NC}=\mathrm{O}), 173.8$ $(\mathrm{OC}=\mathrm{O})$. MS (IE) m/z (\%): $206\left(\mathrm{M}^{+}-121,100\right), 161$ (64), 119 (12), 107 (41) 77 (18), 43 (3). Anal. Calcd. for $\mathrm{C}_{18} \mathrm{H}_{21} \mathrm{~N}_{3} \mathrm{O}_{3}$ (327.38): C 66.04, $\mathrm{H}$ 6.47, N 12.84; Found: $\mathrm{C} 66.28, \mathrm{H} \mathrm{6.60,} \mathrm{N}$ $12.88 \%$.

(S)-1-Phenylethylamonium (S)-5-oxo-1-phenylpyrazolidine-3-carboxylate, (S,S-4). The same procedure described above was followed to afford $7.0 \mathrm{~g}$ ( $85 \%$ yield) of the desired salt $(S, S)-4$ as a white solid, mp $188-190{ }^{\circ} \mathrm{C},[\alpha]_{\mathrm{D}}{ }^{20}=-64.2$ (c 2, MeOH). ${ }^{1} \mathrm{H}-\mathrm{NMR}$ (DMSO- $d_{6}, 400 \mathrm{MHz}$ ): $\delta$ 
$=1.47\left(\mathrm{~d}, J=6.8 \mathrm{~Hz}, 3 \mathrm{H}, \mathrm{CH}_{3}\right), 2.70\left(\mathrm{dd}, J=9.4,16.5 \mathrm{~Hz}, 1 \mathrm{H}, \mathrm{CH}_{2}\right), 2.75(\mathrm{dd}, J=8.7,16.6 \mathrm{~Hz}$, $\left.1 \mathrm{H}, \mathrm{CH}_{2}\right), 3.82\left(\mathrm{dd}, J=9.0,9.2 \mathrm{~Hz}, 1 \mathrm{H},{ }^{*} \mathrm{CH}\right), 4.34\left(\mathrm{q}, J=6.8 \mathrm{~Hz}, 1 \mathrm{H},{ }^{*} \mathrm{CH}\right), 7.03-7.08(\mathrm{~m}, 1 \mathrm{H}$, $\operatorname{ArCH}), 7.30-7.39$ (m, 5H, $\mathrm{ArCH}), 7.47-7.49$ (m, 2H, $\mathrm{ArCH}), 7.79-7.81(\mathrm{~m}, 2 \mathrm{H}, \mathrm{ArCH}) .{ }^{13} \mathrm{C}-$ NMR (DMSO- $\left.d_{6}, 100.5 \mathrm{MHz}\right): \delta=21.9\left(\mathrm{CH}_{3}\right), 39.1\left(\mathrm{CH}_{2}\right), 50.4\left({ }^{*} \mathrm{CH}\right), 57.4\left({ }^{*} \mathrm{CH}\right), 118.2$ (2xArCH), $123.8(\mathrm{ArCH}), 127.2(2 \mathrm{xArCH}), 128.5(\mathrm{ArCH}), 129.0(2 \mathrm{xArCH}), 129.1(2 \mathrm{xArCH})$, $139.7\left(C_{\text {ipso }}\right), 141.2\left(C_{\text {ipso }}\right), 170.9(\mathrm{NC}=\mathrm{O}), 173.8(\mathrm{OC}=\mathrm{O})$. MS (IE) m/z (\%): $206\left(\mathrm{M}^{+}-121,100\right)$, 161 (74), 119 (18), 107 (62), 91 (24), 77 (29), 44 (14). Anal. Calcd. for $\mathrm{C}_{18} \mathrm{H}_{21} \mathrm{~N}_{3} \mathrm{O}_{3}$ (327.38): C 66.04, H 6.47, N 12.84; Found: C 66.34, H 6.23, N 12.96\%.

$(\boldsymbol{R})$-5-Oxo-1-phenylpyrazolidine-3-carboxylic acid, $(\boldsymbol{R}$-3). In a $500 \mathrm{~mL}$ Erlenmeyer flask provided with a magnetic stirrer, $7.0 \mathrm{~g}(21.4 \mathrm{mmol})$ of salt $(R, R)-4$ was dissolved in $200 \mathrm{~mL}$ of water. The $\mathrm{pH}$ was adjusted to 2.0 with $7 \mathrm{~mL}$ of conc. $\mathrm{HCl}$, the solution was placed in an icebath, and was stirred until precipitation of the acid. The precipitate was filtered off, washed with water and dried under vacuum at $40{ }^{\circ} \mathrm{C}$ during $48 \mathrm{~h}$. The desired product $(R)-3(3.9 \mathrm{~g}, 89 \%$ yield $)$ was obtained as a white solid, $\mathrm{mp} 208-210^{\circ} \mathrm{C},[\alpha]_{\mathrm{D}}{ }^{20}=+60.8$ (c 1, MeOH). ${ }^{1} \mathrm{H}-\mathrm{NMR}$ (DMSO$\left.d_{6}, 400 \mathrm{MHz}\right): \delta=2.76\left(\mathrm{dd}, J=5.9,16.1 \mathrm{~Hz}, 1 \mathrm{H}, \mathrm{CH}_{2}\right), 2.98\left(\mathrm{dd}, J=8.6,16.4 \mathrm{~Hz}, 1 \mathrm{H}, \mathrm{CH}_{2}\right)$, $4.23\left(\mathrm{dd}, J=6.1,8.1 \mathrm{~Hz}, 1 \mathrm{H},{ }^{*} \mathrm{CH}\right), 6.55(\mathrm{br}, 1 \mathrm{H}, \mathrm{NH}), 7.06-7.11(\mathrm{~m}, 1 \mathrm{H}, \mathrm{ArCH}), 7.32-7.37$ (m, $2 \mathrm{H}, \mathrm{ArCH}), 7.76-7.79(\mathrm{~m}, 2 \mathrm{H}, \mathrm{ArCH}), 13.0$ (br, $\left.1 \mathrm{H}, \mathrm{CO}_{2} \mathrm{H}\right) .{ }^{13} \mathrm{C}-\mathrm{NMR}\left(\mathrm{DMSO}-d_{6}, 100.5 \mathrm{MHz}\right)$ : $\delta=37.8\left(\mathrm{CH}_{2}\right), 55.4\left({ }^{*} \mathrm{CH}\right), 118.4(2 \mathrm{xArCH}), 124.1(\mathrm{ArCH}), 129.0(2 \mathrm{xArCH}), 139.6\left(C_{\mathrm{ipso}}\right)$, $170.6(\mathrm{NC}=\mathrm{O}), 172.8\left(\mathrm{CO}_{2} \mathrm{H}\right)$. MS (IE) m/z (\%): $206\left(\mathrm{M}^{+}, 100\right), 161$ (56), $118(14), 107$ (61), 93 (20), 77 (19), 55 (5). IR $v_{\max }(\mathrm{KBr}) \mathrm{cm}^{-1}: 3442,3231,2538,1730,1650,1592,1488,1374,1224$, 1008, 763. Anal. Calcd. for $\mathrm{C}_{10} \mathrm{H}_{10} \mathrm{~N}_{2} \mathrm{O}_{3}$ (206.20): C 58.25, H 4.89, N 13.59; Found: C 58.39, H 5.29, N 13.69\%.

(S)-5-Oxo-1-phenylpyrazolidine-3-carboxylic acid, $(S-3)$. The procedure described above (using the same quantities of reagents) was followed to afford the enantiopure carboxylic acid (S)-3 (3.8 g, 87\% yield) as a white solid, $\mathrm{mp} 212-214{ }^{\circ} \mathrm{C},[\alpha]_{\mathrm{D}}{ }^{20}=-59.6(\mathrm{c} 1, \mathrm{MeOH}) .{ }^{1} \mathrm{H}-\mathrm{NMR}$ $\left(\mathrm{DMSO}_{6}, 400 \mathrm{MHz}\right): \delta=2.75\left(\mathrm{dd}, J=5.9,16.6 \mathrm{~Hz}, 1 \mathrm{H}, \mathrm{CH}_{2}\right), 2.98(\mathrm{dd}, J=8.6,16.4 \mathrm{~Hz}, 1 \mathrm{H}$, $\left.\mathrm{CH}_{2}\right), 4.24\left(\mathrm{dd}, J=6.1,8.6 \mathrm{~Hz}, 1 \mathrm{H},{ }^{*} \mathrm{CH}\right), 6.54(\mathrm{br}, 1 \mathrm{H}, \mathrm{NH}), 7.05-7.12(\mathrm{~m}, 1 \mathrm{H}, \mathrm{ArCH}), 7.32-$ $7.38(\mathrm{~m}, 2 \mathrm{H}, \mathrm{ArCH}), 7.76-7.80(\mathrm{~m}, 2 \mathrm{H}, \mathrm{ArCH}), 13.0\left(\mathrm{br}, 1 \mathrm{H}, \mathrm{CO}_{2} \mathrm{H}\right) .{ }^{13} \mathrm{C}-\mathrm{NMR}$ (DMSO-d 6 , $100.5 \mathrm{MHz}): \delta=37.8\left(\mathrm{CH}_{2}\right), 55.4\left({ }^{*} \mathrm{CH}\right), 118.4(2 \mathrm{xArCH}), 124.1(\mathrm{ArCH}), 129.0(2 \mathrm{xArCH})$, $139.6\left(C_{\text {ipso }}\right), 170.6(\mathrm{NC}=\mathrm{O}), 172.8\left(\mathrm{CO}_{2} \mathrm{H}\right) . \mathrm{MS}(\mathrm{IE}) \mathrm{m} / \mathrm{z}(\%): 206\left(\mathrm{M}^{+}, 100\right), 161$ (69), $118(19)$, 107 (56), 93 (22), 77 (24), 55 (6). IR $v_{\max }(\mathrm{KBr}) \mathrm{cm}^{-1}:$ 3454, 3209, 2554, 1740, 1690, 1593, 1494, 1355, 1206, 1018, 753.

(R)-Methyl 5-oxo-1-phenylpyrazolidine-3-carboxylate, $(\boldsymbol{R}-5)$. In a $100 \mathrm{~mL}$ round-bottomed flask equipped with a magnetic stirrer and a reflux condenser, were added $5.0 \mathrm{~g}(24.3 \mathrm{mmol})$ of $(R)-3,80 \mathrm{~mL}$ of methanol, and a catalytic amount of sulfuric acid. The reaction mixture was placed in the microwave equipment and was heated to reflux during 10 min using a power of 50 Watts. The reaction mixture was then concentrated in the rotary evaporator and allowed to cool to RT before the addition of $25 \mathrm{~mL}$ of water. The $\mathrm{pH}$ of the aqueous solution was adjusted to 8.0 by addition of aqueous $\mathrm{NaHCO}_{3}$ to subsequently extract $(3 \times 25 \mathrm{~mL})$ the organic product with ethyl acetate. The organic phase was washed with brine, dried with anhyd. $\mathrm{Na}_{2} \mathrm{SO}_{4}$ and 
concentrated. The concentrate was recrystallised from $7 \mathrm{~mL}$ of hexane-EtOAc $(8: 2 \mathrm{v} / \mathrm{v})$ to give $5.1 \mathrm{~g}(96 \%$ yield $)$ of product $(R)-5$ as a white solid, mp $100-102{ }^{\circ} \mathrm{C},[\alpha]_{\mathrm{D}}{ }^{20}=+88.2(\mathrm{c} 1$, $\left.\mathrm{CHCl}_{3}\right),>99 \%$ ee. ${ }^{1} \mathrm{H}-\mathrm{NMR}\left(\mathrm{CDCl}_{3}, 400 \mathrm{MHz}\right): \delta=2.95\left(\mathrm{dd}, J=9.3,16.7 \mathrm{~Hz}, 1 \mathrm{H}, \mathrm{CH}_{2}\right), 3.00$ $(\mathrm{dd}, 1 \mathrm{H}, J=8.8,16.8 \mathrm{~Hz}), 3.82\left(\mathrm{~s}, 3 \mathrm{H}, \mathrm{CH}_{3}\right), 4.35$ (ddd, $\left.J=8.8,9.2,9.5 \mathrm{~Hz}, 1 \mathrm{H},{ }^{*} \mathrm{CH}\right), 5.27$ (d, $J=9.5 \mathrm{~Hz}, 1 \mathrm{H}, \mathrm{NH}), 7.11-716(\mathrm{~m}, 1 \mathrm{H}, \mathrm{ArCH}), 7.32-7.39(\mathrm{~m}, 2 \mathrm{H}, \mathrm{ArCH}), 7.79-7.84(\mathrm{~m}, 2 \mathrm{H}$, $\operatorname{ArCH}) .{ }^{13} \mathrm{C}-\mathrm{NMR}\left(\mathrm{CDCl}_{3}, 100.5 \mathrm{MHz}\right): \delta=37.9\left(\mathrm{CH}_{2}\right), 52.9\left(\mathrm{CH}_{3}\right), 55.4\left({ }^{*} \mathrm{CH}\right), 118.6$ (2xArCH), $124.7(\mathrm{ArCH}), 128.8(2 \mathrm{xArCH}), 138.3\left(C_{\mathrm{ipso}}\right), 168.3(\mathrm{NC}=\mathrm{O}), 171.3(\mathrm{OC}=\mathrm{O})$. MS (IE) $\mathrm{m} / \mathrm{z}(\%): 220\left(\mathrm{M}^{+}, 100\right), 178$ (4), 161 (62), 118 (6), 107 (8), 91 (2). IR $v_{\max }(\mathrm{KBr}) \mathrm{cm}^{-1}$ : 3446, 3216, 2332, 1742, 1694, 1594, 1494, 1243, 1032, 768. The enantiomeric excess was determined by HPLC using a chiral Chiralpak AD column, $230 \mathrm{~nm}$, mobile phase hexane- $i$ $\mathrm{PrOH}$ (90:10), and flow $1 \mathrm{~mL} / \mathrm{min}$. Retention time: $27.08 \mathrm{~min}(R)$.

(S)-Methyl 5-oxo-1-phenylpyrazolidine-3-carboxylate, $(S-5)$. The procedure described above was followed to produce the desired ester (S)-5, employing $4.6 \mathrm{~g}(22.4 \mathrm{mmol})$ of $(S)-3,75 \mathrm{~mL}$ of methanol and a catalytic amount of sulfuric acid. $4.77 \mathrm{~g}$ of $(S)-5$ were obtained (97\% yield), as a white solid, mp $100-102{ }^{\circ} \mathrm{C},[\alpha]_{\mathrm{D}}{ }^{20}=-89.0\left(\right.$ c $\left.1, \mathrm{CHCl}_{3}\right),>99 \%$ ee. ${ }^{1} \mathrm{H}-\mathrm{NMR}\left(\mathrm{CDCl}_{3}, 400\right.$ $\mathrm{MHz}): \delta=2.94\left(\mathrm{dd}, J=9.3,16.7 \mathrm{~Hz}, 1 \mathrm{H}, \mathrm{CH}_{2}\right), 2.99\left(\mathrm{dd}, J=8.8,16.5 \mathrm{~Hz}, 1 \mathrm{H}, \mathrm{CH}_{2}\right), 3.83(\mathrm{~s}$, $\left.3 \mathrm{H}, \mathrm{CH}_{3}\right), 4.36\left(\mathrm{ddd}, J=8.8,9.2,9.5 \mathrm{~Hz}, 1 \mathrm{H},{ }^{*} \mathrm{CH}\right), 5.27(\mathrm{~d}, J=9.9 \mathrm{~Hz}, 1 \mathrm{H}, \mathrm{NH}), 7.10-7.16(\mathrm{~m}$, $1 \mathrm{H}, \mathrm{ArCH}), 7.32-7.39$ (m, 2H, $\mathrm{ArCH}), 7.79-7.85$ (m, 2H, $\mathrm{ArCH}) .{ }^{13} \mathrm{C}-\mathrm{NMR}\left(\mathrm{CDCl}_{3}, 100.5\right.$ $\mathrm{MHz}): \delta=38.0\left(\mathrm{CH}_{2}\right), 53.0\left(\mathrm{CH}_{3}\right), 55.4\left({ }^{*} \mathrm{CH}\right), 118.6(2 \mathrm{xArCH}), 124.8(\mathrm{ArCH}), 128.9$ (2xArCH), $138.3\left(C_{\text {ipso }}\right), 168.4(\mathrm{NC}=\mathrm{O}), 171.3(\mathrm{OC}=\mathrm{O}) . \mathrm{MS}(\mathrm{IE}) \mathrm{m} / \mathrm{z}(\%): 220\left(\mathrm{M}^{+}, 100\right), 178$ (4), $161(86), 119$ (6), 107 (9), 91 (2). IR $v_{\max }(\mathrm{KBr}) \mathrm{cm}^{-1}:$ 3458, 3216, 2379, 1742, 1694, 1592, 1495, 1241, 1032, 766. Anal. Calcd. for $\mathrm{C}_{11} \mathrm{H}_{12} \mathrm{~N}_{2} \mathrm{O}_{3}$ (220.22): C 59.99, H 5.49, N 12.72; Found:

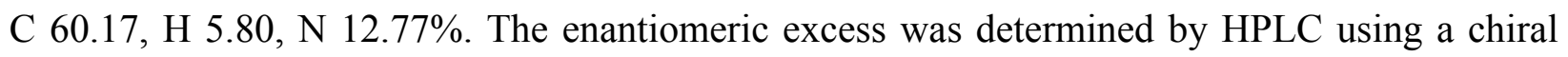
Chiralpak AD column, $230 \mathrm{~nm}$, mobile phase hexane-i-PrOH (90:10), and flow $1 \mathrm{~mL} / \mathrm{min}$. Retention time: $18.95 \min (S)$.

(R)-1-tert-Butyl 5-methyl 3-oxo-2-phenylpyrazolidine-1,5-dicarboxylate, ( $R$-6). In a $250 \mathrm{~mL}$ round-bottomed flask equipped with a magnetic stirrer were placed $4.5 \mathrm{~g}(20.4 \mathrm{mmol})$ of $(R)-5$, $5.0 \mathrm{~g}(40.9 \mathrm{mmol})$ of DMAP and $4.2 \mathrm{~g}(40.9 \mathrm{mmol}, 5.7 \mathrm{~mL})$ of $\mathrm{Et}_{3} \mathrm{~N}$. These reagents were dissolved in $100 \mathrm{~mL}$ of $\mathrm{CH}_{2} \mathrm{Cl}_{2}$ and the resulting mixture was stirred during 10 minutes before the addition of $4.5 \mathrm{~g}$ ( 1 equiv., $20.4 \mathrm{mmol})$ of $(\mathrm{Boc})_{2} \mathrm{O}$. Three additional equivalents of $(\mathrm{Boc})_{2} \mathrm{O}$ were added to the solution - one equivalent each three hours to complete a total of $18.0 \mathrm{~g}$ $(81.7 \mathrm{mmol})$ of $(\mathrm{Boc})_{2} \mathrm{O}$. The reaction mixture was stirred for 39 additional hours after the addition of the fourth equivalent. The solution was extracted three times with $50 \mathrm{~mL}$ of $\mathrm{CH}_{2} \mathrm{Cl}_{2}$ and $50 \mathrm{~mL}$ of water, adjusting the $\mathrm{pH}$ to 4 and washing the organic phase with brine. The organic phase was dried with anhyd. $\mathrm{Na}_{2} \mathrm{SO}_{4}$ and then the $\mathrm{CH}_{2} \mathrm{Cl}_{2}$ solvent was evaporated under vacuum. The crude product was purified by column chromatography (hexane-EtOAc, 85:15 v/v) and recrystallised from hexane-EtOAc $(7: 3 \mathrm{v} / \mathrm{v})$, obtaining $5.4 \mathrm{~g}(83 \%$ yield $)$ of $(R)-6$ as a white solid, mp 98-100 ${ }^{\circ} \mathrm{C},[\alpha]_{\mathrm{D}}{ }^{20}=+61.4\left(\mathrm{c} 1, \mathrm{CHCl}_{3}\right) .{ }^{1} \mathrm{H}-\mathrm{NMR}\left(\mathrm{CDCl}_{3}, 400 \mathrm{MHz}\right): \delta=1.27(\mathrm{~s}, 9 \mathrm{H}$, $\left.\mathrm{C}\left(\mathrm{CH}_{3}\right)_{3}\right), 2.87\left(\mathrm{dd}, J=1.1,17.2 \mathrm{~Hz}, 1 \mathrm{H}, \mathrm{CH}_{2}\right), 3.29\left(\mathrm{dd}, J=9.9,17.2 \mathrm{~Hz}, 1 \mathrm{H}, \mathrm{CH}_{2}\right), 3.81(\mathrm{~s}, 3 \mathrm{H}$, $\left.\mathrm{CH}_{3}\right), 5.22$ (dd, $\left.J=1.1,9.9 \mathrm{~Hz}, 1 \mathrm{H},{ }^{*} \mathrm{CH}\right), 7.14-7.20$ (m, 1H, $\left.\mathrm{ArCH}\right), 7.34-7.40$ (m, 2H, ArCH), 
7.57-7.62 (m, 2H, $\mathrm{ArCH}) .{ }^{13} \mathrm{C}-\mathrm{NMR}\left(\mathrm{CDCl}_{3}, 100.5 \mathrm{MHz}\right): \delta=27.8\left(\mathrm{C}\left(\mathrm{CH}_{3}\right)_{3}\right), 35.6\left(\mathrm{CH}_{2}\right), 53.0$ $\left(\mathrm{CH}_{3}\right), 58.1\left({ }^{*} \mathrm{CH}\right), 83.9\left(\mathrm{C}\left(\mathrm{CH}_{3}\right)_{3}\right), 120.4(2 \times \mathrm{ArCH}), 125.5(\mathrm{ArCH}), 128.6(2 \times \mathrm{ArCH}), 138.9$ $\left(C_{\text {ipso }}\right), 155.3(\mathrm{OC}=\mathrm{ON}), 167.7(\mathrm{NC}=\mathrm{O}), 170.1(\mathrm{OC}=\mathrm{O}) . \mathrm{MS}(\mathrm{IE}) \mathrm{m} / \mathrm{z}(\%): 320\left(\mathrm{M}^{+}, 3\right), 220$ (100), $161(60), 118$ (3), 107 (4), 57 (30). IR $v_{\max }(\mathrm{KBr}) \mathrm{cm}^{-1}: 3426,2980,2345,1762,1741$, 1736, 1594, 1310, 1152, 1022, 757.

(S)-1-tert-Butyl 5-methyl 3-0xo-2-phenylpyrazolidine-1,5-dicarboxylate, (S-6). The procedure described above was followed to obtain the $N$-Boc protected ester (S)-6, employing

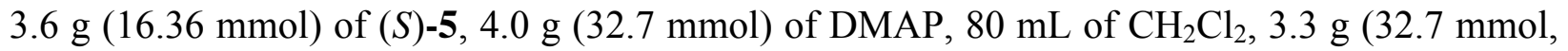
$4.6 \mathrm{~mL})$ of $\mathrm{Et}_{3} \mathrm{~N}$ and $14.4 \mathrm{~g}(65.4 \mathrm{mmol})$ of $(\mathrm{Boc})_{2} \mathrm{O}$. The desired product $4.5 \mathrm{~g}$ of $(S)-6(4.5 \mathrm{~g}$, $87 \%$ yield) was obtained as a white solid, mp $98-100{ }^{\circ} \mathrm{C},[\alpha]_{\mathrm{D}}{ }^{20}=-62.1\left(\mathrm{c} 1, \mathrm{CHCl}_{3}\right) .{ }^{1} \mathrm{H}-\mathrm{NMR}$ $\left(\mathrm{CDCl}_{3}, 400 \mathrm{MHz}\right): \delta=1.26\left(\mathrm{~s}, 9 \mathrm{H}, \mathrm{C}\left(\mathrm{CH}_{3}\right)_{3}\right), 2.87\left(\mathrm{dd}, J=1.3,17.0 \mathrm{~Hz}, 1 \mathrm{H}, \mathrm{CH}_{2}\right), 3.30(\mathrm{dd}, J=$ 9.7, $\left.17.0 \mathrm{~Hz}, 1 \mathrm{H}, \mathrm{CH}_{2}\right), 3.80\left(\mathrm{~s}, 3 \mathrm{H}, \mathrm{CH}_{3}\right), 5.22\left(\mathrm{dd}, J=1.1,9.5 \mathrm{~Hz}, 1 \mathrm{H},{ }^{*} \mathrm{CH}\right), 7.14-7.20(\mathrm{~m}$, $1 \mathrm{H}, \mathrm{ArCH}), 7.34-7.39(\mathrm{~m}, 2 \mathrm{H}, \mathrm{ArCH}), 7.57-7.62(\mathrm{~m}, 2 \mathrm{H}, \mathrm{ArCH}) .{ }^{13} \mathrm{C}-\mathrm{NMR}\left(\mathrm{CDCl}_{3}, 100.5\right.$

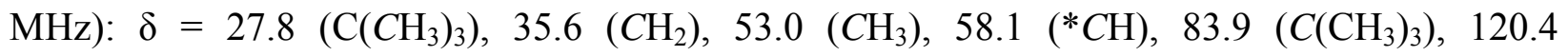
$(2 \times \mathrm{ArCH}), 125.5(\mathrm{ArCH}), 128.6(2 \times \mathrm{ArCH}), 138.9\left(C_{\mathrm{ipso}}\right), 155.3(\mathrm{OC}=\mathrm{ON}), 167.7(\mathrm{NC}=\mathrm{O}), 170.2$ $(\mathrm{OC}=\mathrm{O})$. MS (IE) m/z (\%): $320\left(\mathrm{M}^{+}, 8\right), 220$ (100), 161 (59), 118 (4), 107 (5), 77 (3), 57 (27). IR $v_{\max }(\mathrm{KBr}) \mathrm{cm}^{-1}: 3426,2980,2345,1762,1741,1736,1594,1310,1152,1022,757$. Anal. Calcd. for $\mathrm{C}_{16} \mathrm{H}_{20} \mathrm{~N}_{2} \mathrm{O}_{5}$ (320.34): C 59.99, H 6.29, N 8.74; Found: C 60.37, H 6.69, N 8.75\%.

(R)-2-(tert-Butoxycarbonyl)-5-oxo-1-phenylpyrazolidine-3-carboxylic acid, (R-7). In a 500 $\mathrm{mL}$ round-bottomed flask equipped with a magnetic stirrer were placed $5.27 \mathrm{~g}(16.45 \mathrm{mmol})$ of $(R)-6,100 \mathrm{~mL}$ of THF and $1.03 \mathrm{~g}(24.7 \mathrm{mmol})$ of $\mathrm{LiOH} \cdot \mathrm{H}_{2} \mathrm{O}$ and the reaction mixture was stirred during $44 \mathrm{~h}$ at room temperature. The solvent was evaporated and $50 \mathrm{~mL}$ of EtOAc was added before extracting with $100 \mathrm{~mL}$ of a saturated aqueous solution of $\mathrm{NaHCO}_{3}$. The organic phase was extracted again with $90 \mathrm{~mL}$ of water. The $\mathrm{pH}$ of the aqueous phase was adjusted to 3.0 with a saturated solution of tartaric acid and extracted five times with $50 \mathrm{~mL}$ of EtOAc and the organic phase was washed with brine, dried with anhyd. $\mathrm{Na}_{2} \mathrm{SO}_{4}$ and concentrated under vacuum. The crude product was purified by column chromatography $\left(\mathrm{CHCl}_{3}-\mathrm{MeOH}, 95: 5 \mathrm{v} / \mathrm{v}\right)$ to afford $4.51 \mathrm{~g}\left(90 \%\right.$ yield) of $(R)-7$ as a white solid, $\mathrm{mp} 138-140{ }^{\circ} \mathrm{C},[\alpha]_{\mathrm{D}}{ }^{20}=+49.9\left(\mathrm{c} 1, \mathrm{CHCl}_{3}\right)$. ${ }^{1} \mathrm{H}$ - NMR (DMSO- $\left.d_{6}, 400 \mathrm{MHz}, 120^{\circ} \mathrm{C}\right): \delta=1.30\left(\mathrm{~s}, 9 \mathrm{H}, \mathrm{C}\left(\mathrm{CH}_{3}\right)_{3}\right), 2.67(\mathrm{dd}, J=1.3,17.0 \mathrm{~Hz}$, $\left.1 \mathrm{H}, \mathrm{CH}_{2}\right), 3.37$ (dd, $\left.J=9.3,17.0 \mathrm{~Hz}, 1 \mathrm{H}, \mathrm{CH}_{2}\right), 5.05\left(\mathrm{dd}, J=1.2,9.6 \mathrm{~Hz}, 1 \mathrm{H},{ }^{*} \mathrm{CH}\right), 7.12-7.18$ $(\mathrm{m}, 1 \mathrm{H}, \mathrm{ArCH}), 7.32-7.39(\mathrm{~m}, 2 \mathrm{H}, \mathrm{ArCH}), 7.50-7.56(\mathrm{~m}, 2 \mathrm{H}, \mathrm{ArCH}) .{ }^{13} \mathrm{C}-\mathrm{NMR}$ (DMSO- $d_{6}$,

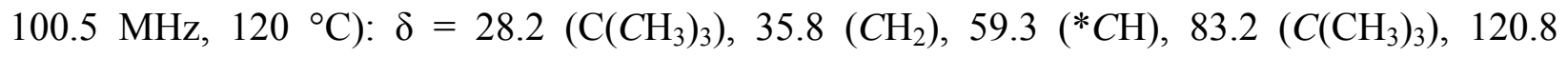
$(2 \mathrm{xArCH}), 125.3(\mathrm{ArCH}), 128.7(2 \mathrm{xArCH}), 140.0\left(C_{\mathrm{ipso}}\right), 155.5(\mathrm{OC}=\mathrm{ON}), 169.5(\mathrm{NC}=\mathrm{O}), 171.7$ $\left(\mathrm{CO}_{2} \mathrm{H}\right)$. MS (IE) $\mathrm{m} / \mathrm{z}(\%): 306\left(\mathrm{M}^{+}, 2\right), 206$ (47), 161 (35), 107 (11), 77 (8), 57 (100), 41 (17). IR $v_{\max }(\mathrm{KBr}) \mathrm{cm}^{-1}: 3440,2980,2624,1742,1728,1598,1317,1156,753$. HRMS (ESI-TOF) calculated for $\mathrm{C}_{15} \mathrm{H}_{18} \mathrm{~N}_{2} \mathrm{O}_{5}+\mathrm{H}^{+}$: 307.1288; Found: 307.1288 .

(S)-2-(tert-Butoxycarbonyl)-5-oxo-1-phenylpyrazolidine-3-carboxylic acid, (S-7). The procedure described above was followed with $4.87 \mathrm{~g}(15.21 \mathrm{mmol})$ of $(S)-6,100 \mathrm{~mL}$ of THF and $0.96 \mathrm{~g}(22.8 \mathrm{mmol})$ of $\mathrm{LiOH} \cdot \mathrm{H}_{2} \mathrm{O}$ to afford the desired $N$-Boc protected carboxylic acid $(\mathrm{S})-7$ in $89 \%$ yield $(4.15 \mathrm{~g})$ as a white solid, mp $136-138{ }^{\circ} \mathrm{C},[\alpha]_{\mathrm{D}}{ }^{20}=-50.1\left(\right.$ c $\left.1, \mathrm{CHCl}_{3}\right) .{ }^{1} \mathrm{H}-\mathrm{NMR}$ 
$\left(\mathrm{DMSO}_{6}, 400 \mathrm{MHz}, 120^{\circ} \mathrm{C}\right): \delta=1.30\left(\mathrm{~s}, 9 \mathrm{H}, \mathrm{C}\left(\mathrm{CH}_{3}\right)_{3}\right), 2.68\left(\mathrm{dd}, J=1.4,17.0 \mathrm{~Hz}, 1 \mathrm{H}, \mathrm{CH}_{2}\right)$, $3.39\left(\mathrm{dd}, J=9.4,16.9 \mathrm{~Hz}, 1 \mathrm{H}, \mathrm{CH}_{2}\right), 5.07\left(\mathrm{dd}, J=1.5,9.4 \mathrm{~Hz}, 1 \mathrm{H},{ }^{*} \mathrm{CH}\right), 7.13-7.19(\mathrm{~m}, 1 \mathrm{H}$, $\mathrm{ArCH}), 7.30-7.39$ (m, 2H, ArCH), 7.48-7.55 (m, 2H, ArCH). ${ }^{13} \mathrm{C}-\mathrm{NMR}$ (DMSO-d $6,100.5 \mathrm{MHz}$, $\left.120{ }^{\circ} \mathrm{C}\right): \delta=28.1\left(\mathrm{C}\left(\mathrm{CH}_{3}\right)_{3}\right), 35.7\left(\mathrm{CH}_{2}\right), 59.1\left({ }^{*} \mathrm{CH}\right), 83.3\left(\mathrm{C}\left(\mathrm{CH}_{3}\right)_{3}\right), 121.0(2 \mathrm{xArCH}), 125.3$ $(\mathrm{ArCH}), 128.8(2 \times \mathrm{xArCH}), 139.9\left(C_{\mathrm{ipso}}\right), 155.5(\mathrm{OC}=\mathrm{ON}), 169.4(\mathrm{NC}=\mathrm{O}), 171.5\left(\mathrm{CO}_{2} \mathrm{H}\right) . \mathrm{MS}(\mathrm{IE})$ $\mathrm{m} / \mathrm{z}(\%): 306\left(\mathrm{M}^{+}, 1\right), 206$ (43), 161 (32), 107 (12), 77 (8), 57 (100). IR $v_{\max }(\mathrm{KBr}) \mathrm{cm}^{-1}: 3446$, 2980, 2624, 1742, 1728, 1598, 1317, 1156, 748.

\section{General procedure for the preparation of amides 8-11}

The required quantity of the corresponding precursor, the $N$-Boc protected carboxylic acid was dissolved with dry THF in a round-bottomed flask equipped with a magnetic stirrer and an addition funnel. An equimolar amount of $\mathrm{Et}_{3} \mathrm{~N}$ was added to the solution and the resulting mixture was stirred for ten minutes. At this moment, the flask was placed in an ice-bath and one equivalent of pivaloyl chloride was added slowly during approximately ten minutes. The reaction mixture was stirred at $0{ }^{\circ} \mathrm{C}$ during $1 \mathrm{~h}$, and then one equivalent of the corresponding amine was added. The flask was subsequently set inside the microwave equipment and the reaction mixture was heated at $70{ }^{\circ} \mathrm{C}$ during 30 minutes, using a power of 75 Watts. Afterwards, the solvent was evaporated and the crude product was extracted with EtOAc, the organic phase was washed with brine, dried with $\mathrm{Na}_{2} \mathrm{SO}_{4}$ and concentrated under vacuum. The product was recrystallised from hexane-EtOAc $(9: 1 \mathrm{v} / \mathrm{v})$, filtered and dried at $40{ }^{\circ} \mathrm{C}$ under vacuum.

(R)-tert-Butyl 3-oxo-2-phenyl-5-(phenylcarbamoyl)pyrazolidine-1-carboxylate, $(\boldsymbol{R}-8)$. The General Procedure described above was followed with $0.6 \mathrm{~g}(1.96 \mathrm{mmol})$ of $(R)-7,0.21 \mathrm{~g}$ $(2 \mathrm{mmol}, 0.29 \mathrm{~mL})$ of $\mathrm{Et}_{3} \mathrm{~N}, 0.25 \mathrm{~g}(2 \mathrm{mmol}, 0.25 \mathrm{~mL})$ of pivaloyl chloride, $0.19 \mathrm{~g}(2 \mathrm{mmol}$, $0.19 \mathrm{~mL})$ of aniline and $40 \mathrm{~mL}$ of THF to afford the desired $N$-Boc protected amide $(R)-8$, $(0.62 \mathrm{~g}, 83 \%$ yield $)$ as a white solid, $\mathrm{mp} 178-180{ }^{\circ} \mathrm{C},[\alpha]_{\mathrm{D}}{ }^{20}=+13.7\left(\mathrm{c} 1, \mathrm{CHCl}_{3}\right) .{ }^{1} \mathrm{H}-\mathrm{NMR}$ $\left(\mathrm{CDCl}_{3}, 400 \mathrm{MHz}\right): \delta=1.33\left(\mathrm{~s}, 9 \mathrm{H}, \mathrm{C}\left(\mathrm{CH}_{3}\right)_{3}\right), 3.20\left(\mathrm{dd}, J=9.7,17.4 \mathrm{~Hz}, 1 \mathrm{H}, \mathrm{CH}_{2}\right), 3.36(\mathrm{dd}, J=$ 1.6, 17.4 Hz, 1H, $\left.\mathrm{CH}_{2}\right), 5.13\left(\mathrm{dd}, J=1.6,9.7 \mathrm{~Hz}, 1 \mathrm{H},{ }^{*} \mathrm{CH}\right), 7.13-7.23(\mathrm{~m}, 2 \mathrm{H}, \mathrm{ArCH}), 7.32-7.42$ $(\mathrm{m}, 4 \mathrm{H}, \mathrm{ArCH}), 7.51-7.57(\mathrm{~m}, 4 \mathrm{H}, \mathrm{ArCH}), 8.56$ (br, $1 \mathrm{H}, \mathrm{NH}) .{ }^{13} \mathrm{C}-\mathrm{NMR}\left(\mathrm{CDCl}_{3}, 100.5 \mathrm{MHz}\right): \delta$ $=27.8\left(\mathrm{C}_{\left.\left(\mathrm{CH}_{3}\right)_{3}\right),} 33.8\left(\mathrm{CH}_{2}\right), 60.0\left({ }^{*} \mathrm{CH}\right), 85.2\left(\mathrm{C}\left(\mathrm{CH}_{3}\right)_{3}\right), 119.7(2 \mathrm{xArCH}), 120.1(2 \mathrm{xArCH})\right.$, $125.1(\mathrm{ArCH}), 125.7(\mathrm{ArCH}), 129.0(2 \mathrm{xArCH}), 129.2(2 \mathrm{xArCH}), 137.1\left(C_{\mathrm{ipso}}\right), 138.8\left(C_{\mathrm{ipso}}\right)$, $156.6(\mathrm{OC}=\mathrm{ON}), 166.7\left(\mathrm{NC}=\mathrm{OCH}_{2}\right), 168.3(* \mathrm{CHC}=\mathrm{ON}) . \mathrm{MS}(\mathrm{IE}) \mathrm{m} / \mathrm{z}(\%): 381\left(\mathrm{M}^{+}, 2\right), 281$ (77), 161 (100), 119 (7), 93 (14), 57 (52). IR $v_{\max }(\mathrm{KBr}) \mathrm{cm}^{-1}:$ 3446, 3231, 1733, 1697, 1677, 1516, 1316, 1158, 763. Anal. Calcd. for $\mathrm{C}_{21} \mathrm{H}_{23} \mathrm{~N}_{3} \mathrm{O}_{4}$ (381.43): C 66.13, $\mathrm{H}$ 6.08, N 11.02; Found: C 65.84, H 6.19, N 11.12\%.

(R)-tert-Butyl 5-(benzylcarbamoyl)-3-oxo-2-phenylpyrazolidine-1-carboxylate, $(\boldsymbol{R}-9)$. The General Procedure was followed with $0.6 \mathrm{~g}(1.96 \mathrm{mmol})$ of $(R)-7,0.21 \mathrm{~g}(2 \mathrm{mmol}, 0.29 \mathrm{~mL})$ of $\mathrm{Et}_{3} \mathrm{~N}, 0.25 \mathrm{~g}(2 \mathrm{mmol}, 0.25 \mathrm{~mL})$ of pivaloyl chloride, $0.22 \mathrm{~g}(2 \mathrm{mmol}, 0.22 \mathrm{~mL})$ of benzylamine and $40 \mathrm{~mL}$ of THF to obtain the $N$-Boc protected amide $(R)-9,(0.49 \mathrm{~g}, 63 \%$ yield) as a white solid, mp 162-164 ${ }^{\circ} \mathrm{C},[\alpha]_{\mathrm{D}}{ }^{20}=-7.5\left(\mathrm{c} 1, \mathrm{CHCl}_{3}\right) .{ }^{1} \mathrm{H}-\mathrm{NMR}\left(\mathrm{CDCl}_{3}, 400 \mathrm{MHz}\right): \delta=1.28(\mathrm{~s}, 9 \mathrm{H}$, $\left.\mathrm{C}\left(\mathrm{CH}_{3}\right)_{3}\right), 3.15\left(\mathrm{dd}, J=9.9,17.2 \mathrm{~Hz}, 1 \mathrm{H}, \mathrm{CH}_{2}\right), 3.31\left(\mathrm{dd}, J=1.5,17.2 \mathrm{~Hz}, 1 \mathrm{H}, \mathrm{CH}_{2}\right), 4.41$ (dd, $J$ 
$\left.=5.1,14.7 \mathrm{~Hz}, 1 \mathrm{H}, \mathrm{CH}_{2} \mathrm{Ph}\right), 4.60\left(\mathrm{dd}, J=6.6,14.7 \mathrm{~Hz}, 1 \mathrm{H}, \mathrm{CH}_{2} \mathrm{Ph}\right), 5.05(\mathrm{dd}, J=1.5,9.6 \mathrm{~Hz}$, $\left.1 \mathrm{H},{ }^{*} \mathrm{CH}\right), 7.00(\mathrm{br}, 1 \mathrm{H}, \mathrm{NH}), 7.13-7.24(\mathrm{~m}, 3 \mathrm{H}, \mathrm{ArCH}), 7.26-7.33$ (m, 5H, ArCH), 7.39-7.43 (m,

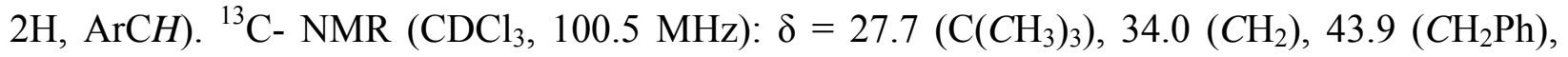

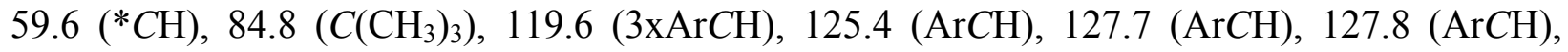
$128.7(2 \mathrm{xArCH}), 128.8(2 \mathrm{xArCH}), 137.4\left(C_{\mathrm{ipso}}\right), 138.6\left(C_{\mathrm{ipso}}\right), 156.5(\mathrm{OC}=\mathrm{ON}), 168.4$ $\left(\mathrm{NC}=\mathrm{OCH}_{2}\right), 168.6\left({ }^{*} \mathrm{CHC}=\mathrm{ON}\right) . \mathrm{MS}(\mathrm{IE}) \mathrm{m} / \mathrm{z}(\%): 395\left(\mathrm{M}^{+}, 1\right), 295$ (73), 189 (7), 161 (100), 105 (11), 91 (17), 57 (46), 41 (7). IR $v_{\max }(\mathrm{KBr}) \mathrm{cm}^{-1}:$ 3419, 3280, 1721, 1708, 1678, 1534, 1317, 1159, 746. Anal. Calcd. for $\mathrm{C}_{22} \mathrm{H}_{25} \mathrm{~N}_{3} \mathrm{O}_{4}$ (395.45): C 66.82, H 6.37, N 10.63; Found: C 67.21, H 6.24, N 10.70\%.

(R)-tert-Butyl 3-oxo-2-phenyl-5-[(S)-1-phenylethylcarbamoyl]pyrazolidine-1-carbo-xylate $(\boldsymbol{R}, \boldsymbol{S}-10)$. The General Procedure was followed with $0.6 \mathrm{~g}(1.96 \mathrm{mmol})$ of $(R)-7,0.21 \mathrm{~g}(2 \mathrm{mmol}$, $0.29 \mathrm{~mL}$ ) of $\mathrm{Et}_{3} \mathrm{~N}, 0.25 \mathrm{~g}$ ( $\left.2 \mathrm{mmol}, 0.25 \mathrm{~mL}\right)$ of pivaloyl chloride, $0.25 \mathrm{~g}$ ( $\left.2 \mathrm{mmol}, 0.26 \mathrm{~mL}\right)$ of (S)- $\alpha$-methylbenzylamine and $40 \mathrm{~mL}$ of THF to obtain the $N$-Boc protected amide $(R, S)-10$ $\left(0.63 \mathrm{~g}, 79 \%\right.$ yield) as a white solid, mp $172-174{ }^{\circ} \mathrm{C},[\alpha]_{\mathrm{D}}{ }^{20}=-46.2$ (c 1, MeOH). ${ }^{1} \mathrm{H}-\mathrm{NMR}$ $\left(\mathrm{CDCl}_{3}, 400 \mathrm{MHz}\right): \delta=1.28\left(\mathrm{~s}, 9 \mathrm{H}, \mathrm{C}\left(\mathrm{CH}_{3}\right)_{3}\right), 1.55\left(\mathrm{~d}, J=7.0 \mathrm{~Hz}, 3 \mathrm{H}, * \mathrm{CHCH}_{3}\right), 3.09(\mathrm{dd}, J=$ 9.7, $\left.17.4 \mathrm{~Hz}, 1 \mathrm{H}, \mathrm{CH}_{2}\right), 3.28\left(\mathrm{dd}, J=1.8,17.4 \mathrm{~Hz}, 1 \mathrm{H}, \mathrm{CH}_{2}\right), 5.01(\mathrm{dd}, 1 \mathrm{H}, J=1.7,9.7 \mathrm{~Hz}), 5.13$ $\left(\mathrm{dq}, J=7.2,7.9 \mathrm{~Hz}, 1 \mathrm{H}, \mathrm{NH}^{*} \mathrm{CHCH}_{3}\right), 6.96(\mathrm{~d}, J=8.1 \mathrm{~Hz}, 1 \mathrm{H}, \mathrm{NH}), 7.10-7.15(\mathrm{~m}, 1 \mathrm{H}, \mathrm{ArCH})$,

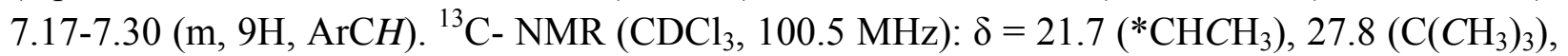

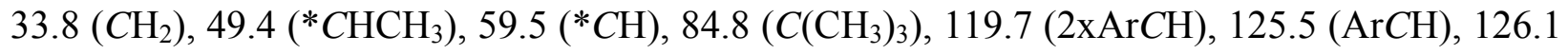
$(2 \mathrm{xArCH}), 127.7(\mathrm{ArCH}), 128.8(2 \times \mathrm{ArCH}), 128.9(2 \times \mathrm{ArCH}), 138.6\left(C_{\mathrm{ipso}}\right), 142.5\left(C_{\mathrm{ipso}}\right), 156.4$ $(\mathrm{OC}=\mathrm{ON}), 167.7\left(\mathrm{NC}=\mathrm{OCH}_{2}\right), 168.6\left({ }^{*} \mathrm{CHC}=\mathrm{ON}\right) . \mathrm{MS}(\mathrm{IE}) \mathrm{m} / \mathrm{z}(\%): 409\left(\mathrm{M}^{+}, 2\right), 309(53), 205$ (42), 161 (100), 120 (5), 105 (85), 77 (7), 57 (58). IR $v_{\max }(\mathrm{KBr}) \mathrm{cm}^{-1}:$ 3419, 3318, 1723, 1703, 1677, 1522, 1314, 1158, 755. Anal. Calcd. for $\mathrm{C}_{23} \mathrm{H}_{27} \mathrm{~N}_{3} \mathrm{O}_{4}$ (409.48): C 67.46, H 6.65, N 10.26; Found: C 67.13, H 6.87, N 10.35\%.

(R)-tert-Butyl 5-[(S)-1-hydroxybutan-2-ylcarbamoyl]-3-oxo-2-phenylpyrazolidine-1carboxylate, $(\boldsymbol{R}, \boldsymbol{S}-\mathbf{1 1})$. The General Procedure was followed with $0.6 \mathrm{~g}(1.96 \mathrm{mmol})$ of $(R)-7$, $0.21 \mathrm{~g}(2 \mathrm{mmol}, 0.29 \mathrm{~mL})$ of $\mathrm{Et}_{3} \mathrm{~N}, 0.25 \mathrm{~g}(2 \mathrm{mmol}, 0.25 \mathrm{~mL})$ of pivaloyl chloride, $0.18 \mathrm{~g}$ ( $2 \mathrm{mmol}, 0.2 \mathrm{~mL}$ ) of $(S)$-2-amino-1-butanol and $40 \mathrm{~mL}$ of THF to obtain the $N$-Boc protected amide $(R, S)-11(0.58 \mathrm{~g}, 79 \%$ yield $)$ as a white solid, mp $180-182{ }^{\circ} \mathrm{C},[\alpha]_{\mathrm{D}}{ }^{20}=+6.6(\mathrm{c} 1, \mathrm{MeOH})$. ${ }^{1} \mathrm{H}-\mathrm{NMR}\left(\mathrm{CDCl}_{3}+\mathrm{DMSO}_{6} 5: 1 \mathrm{v} / \mathrm{v}, 300 \mathrm{MHz}\right): \delta=0.83\left(\mathrm{t}, J=7.5 \mathrm{~Hz}, 3 \mathrm{H}, \mathrm{CH}_{2} \mathrm{CH}_{3}\right), 1.21(\mathrm{~s}$, 9H, $\left.\mathrm{C}\left(\mathrm{CH}_{3}\right)_{3}\right), 1.39-1.62\left(\mathrm{~m}, 2 \mathrm{H}, \mathrm{CH}_{2} \mathrm{CH}_{3}\right), 2.87$ (d, $\left.J=17.1 \mathrm{~Hz}, 1 \mathrm{H}, \mathrm{CH}_{2}\right), 3.12$ (dd, J = 9.6, $\left.17.1 \mathrm{~Hz}, 1 \mathrm{H}, \mathrm{CH}_{2}\right), 3.43\left(\mathrm{dd}, J=4.3,10.9 \mathrm{~Hz}, 1 \mathrm{H},{ }^{*} \mathrm{CHCH}_{2} \mathrm{OH}\right), 4.49(\mathrm{dd}, 4.3,10.9 \mathrm{~Hz}, 1 \mathrm{H}$, $\left.{ }^{*} \mathrm{CHCH}_{2} \mathrm{OH}\right), 3.65-3.79\left(\mathrm{~m}, 1 \mathrm{H},{ }^{*} \mathrm{CHCH}_{2} \mathrm{OH}\right), 4.97\left(\mathrm{~d}, J=9.0 \mathrm{~Hz}, 1 \mathrm{H},{ }^{*} \mathrm{CH}\right), 7.03-7.10(\mathrm{~m}, 1 \mathrm{H}$, $\operatorname{ArCH}), 7.23-7.29(\mathrm{~m}, 2 \mathrm{H}, \mathrm{ArCH}), 7.39(\mathrm{~d}, J=8.4 \mathrm{~Hz}, \mathrm{NH}), 7.45-7.48(\mathrm{~m}, 2 \mathrm{H}, \mathrm{ArCH}) .{ }^{13} \mathrm{C}-$ NMR $\left(\mathrm{CDCl}_{3}+\right.$ DMSO-d $\left._{6} 5: 1 \mathrm{v} / \mathrm{v}, 75.5 \mathrm{MHz}\right): \delta=10.6\left(\mathrm{CH}_{2} \mathrm{CH}_{3}\right), 24.1\left(\mathrm{CH}_{2} \mathrm{CH}_{3}\right), 27.8$ $\left(\mathrm{C}\left(\mathrm{CH}_{3}\right)_{3}\right), 34.8\left(\mathrm{CH}_{2}\right), 52.9\left({ }^{*} \mathrm{CHCH}_{2} \mathrm{OH}\right), 59.0(* \mathrm{CH}), 63.0\left({ }^{*} \mathrm{CHCH}_{2} \mathrm{OH}\right), 83.7\left(\mathrm{C}_{\left.\left(\mathrm{CH}_{3}\right)_{3}\right),}\right.$ $120.5(2 \mathrm{xArCH}), 125.3(\mathrm{ArCH}), 128.5(2 \mathrm{xArCH}), 138.8\left(C_{\mathrm{ipso}}\right), 155.8(\mathrm{OC}=\mathrm{ON}), 168.5$ $\left(\mathrm{NC}=\mathrm{OCH}_{2}\right), 168.8\left({ }^{*} \mathrm{CHC}=\mathrm{ON}\right) . \mathrm{MS}(\mathrm{IE}) \mathrm{m} / \mathrm{z}(\%): 377\left(\mathrm{M}^{+}, 2\right), 277$ (46), 171 (26), 161 (100), 108 (14), 57 (56), 41 (8). IR $v_{\max }(\mathrm{KBr}) \mathrm{cm}^{-1}:$ 3496, 3320, 2935, 1736, 1709, 1684, 1547, 1307 , 
1154, 1030, 761. Anal. Calcd. for $\mathrm{C}_{19} \mathrm{H}_{27} \mathrm{~N}_{3} \mathrm{O}_{5}$ (377.43): C 60.46, H 7.21, N 11.13; Found: C $60.70, \mathrm{H} 7.59$, N 11.29\%.

(S)-tert-Butyl 3-oxo-2-phenyl-5-(phenylcarbamoyl)pyrazolidine-1-carboxylate, (S-8). The General Procedure was followed with $0.55 \mathrm{~g}(1.79 \mathrm{mmol})$ of $(S)-7,0.19 \mathrm{~g}(1.88 \mathrm{mmol}, 0.27 \mathrm{~mL})$ of $\mathrm{Et}_{3} \mathrm{~N}, 0.23 \mathrm{~g}$ (1.88 mmol, $\left.0.23 \mathrm{~mL}\right)$ of pivaloyl chloride, $0.17 \mathrm{~g}(1.88 \mathrm{mmol}, 0.17 \mathrm{~mL})$ of aniline and $35 \mathrm{~mL}$ of THF to obtain the $N$-Boc protected amide $(S)-8(0.6 \mathrm{~g}, 88 \%$ yield $)$ as a white solid, $\mathrm{mp} 176-178{ }^{\circ} \mathrm{C},[\alpha]_{\mathrm{D}}{ }^{20}=-14.2\left(\mathrm{c} 1, \mathrm{CHCl}_{3}\right) .{ }^{1} \mathrm{H}-\mathrm{NMR}\left(\mathrm{CDCl}_{3}, 400 \mathrm{MHz}\right): \delta=1.32$ $\left(\mathrm{s}, 9 \mathrm{H}, \mathrm{C}\left(\mathrm{CH}_{3}\right)_{3}\right), 3.19\left(\mathrm{dd}, J=9.7,17.4 \mathrm{~Hz}, 1 \mathrm{H}, \mathrm{CH}_{2}\right), 3.36\left(\mathrm{dd}, J=1.8,17.6 \mathrm{~Hz}, 1 \mathrm{H}, \mathrm{CH}_{2}\right), 5.13$ $\left(\mathrm{dd}, J=1.7,9.7 \mathrm{~Hz}, 1 \mathrm{H},{ }^{*} \mathrm{CH}\right), 7.12-7.22(\mathrm{~m}, 2 \mathrm{H}, \mathrm{ArCH}), 7.31-7.42$ (m, 4H, ArCH), 7.49-7.52

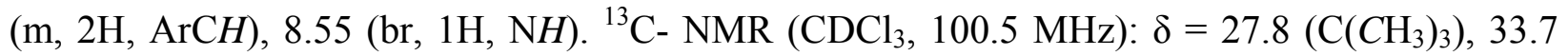
$\left(\mathrm{CH}_{2}\right), 59.9\left({ }^{*} \mathrm{CH}\right), 85.1\left(\mathrm{C}\left(\mathrm{CH}_{3}\right)_{3}\right), 119.6(2 \mathrm{xArCH}), 120.0(2 \mathrm{xArCH}), 125.0(\mathrm{ArCH}), 125.6$ $(\mathrm{ArCH}), 128.9$ (2xArCH), $129.2(2 \times \mathrm{ArCH}), 137.1\left(C_{\mathrm{ipso}}\right), 138.8\left(C_{\mathrm{ipso}}\right), 156.5(\mathrm{OC}=\mathrm{ON}), 166.7$ $\left(\mathrm{NC}=\mathrm{OCH}_{2}\right), 168.2(\mathrm{C}=\mathrm{ONPh}) . \mathrm{MS}(\mathrm{IE}) \mathrm{m} / \mathrm{z}(\%): 381\left(\mathrm{M}^{+}, 1\right), 281$ (75), 175 (6), 161 (100), 119 (5), 93 (11), 77 (5), 57 (62). IR $v_{\max }(\mathrm{KBr}) \mathrm{cm}^{-1}:$ 3446, 3231, 1733, 1697, 1678, 1516, 1316, $1158,763$.

(S)-tert-Butyl 5-(benzylcarbamoyl)-3-oxo-2-phenylpyrazolidine-1-carboxylate, (S-9). The General Procedure was followed with $0.55 \mathrm{~g}(1.79 \mathrm{mmol})$ of $(S)-7,0.19 \mathrm{~g}(1.88 \mathrm{mmol}, 0.27 \mathrm{~mL})$ of $\mathrm{Et}_{3} \mathrm{~N}, 0.23 \mathrm{~g}$ (1.88 mmol, $\left.0.23 \mathrm{~mL}\right)$ of pivaloyl chloride, $0.20 \mathrm{~g}$ (1.88 mmol, $\left.0.20 \mathrm{~mL}\right)$ of benzylamine and $35 \mathrm{~mL}$ of THF to obtain the $N$-Boc protected amide $(S)-9(0.57 \mathrm{~g}, 81 \%$ yield $)$ as a white solid, mp 158-160 ${ }^{\circ} \mathrm{C},[\alpha]_{\mathrm{D}}{ }^{20}=+8.0\left(\mathrm{c} 1, \mathrm{CHCl}_{3}\right) .{ }^{1} \mathrm{H}-\mathrm{NMR}\left(\mathrm{CDCl}_{3}, 400 \mathrm{MHz}\right): \delta=$ $1.28\left(\mathrm{~s}, 9 \mathrm{H}, \mathrm{C}\left(\mathrm{CH}_{3}\right)_{3}\right), 3.15\left(\mathrm{dd}, J=9.9,17.2 \mathrm{~Hz}, 1 \mathrm{H}, \mathrm{CH}_{2}\right), 3.32\left(\mathrm{dd}, J=1.5,17.2 \mathrm{~Hz}, 1 \mathrm{H}, \mathrm{CH}_{2}\right)$, $4.41\left(\mathrm{dd}, J=5.1,14.6 \mathrm{~Hz}, 1 \mathrm{H}, \mathrm{CH}_{2} \mathrm{Ph}\right), 4.61\left(\mathrm{dd}, J=6.6,14.6 \mathrm{~Hz}, 1 \mathrm{H}, \mathrm{CH}_{2} \mathrm{Ph}\right), 5.05(\mathrm{dd}, J=$ 1.6, 9.7 Hz, 1H, *CH), 7.00 (br, $1 \mathrm{H}, \mathrm{NH}), 7.13-7.34$ (m, 8H, $\mathrm{ArCH}), 7.38-7.43$ (m, 2H, ArCH). ${ }^{13} \mathrm{C}-\mathrm{NMR}\left(\mathrm{CDCl}_{3}, 100.5 \mathrm{MHz}\right): \delta=27.8\left(\mathrm{C}\left(\mathrm{CH}_{3}\right)_{3}\right), 34.1\left(\mathrm{CH}_{2}\right), 44.0\left(\mathrm{CH}_{2} \mathrm{Ph}\right), 59.7(* \mathrm{CH})$, $85.0\left(\mathrm{C}\left(\mathrm{CH}_{3}\right)_{3}\right), 119.6(2 \mathrm{xArCH}), 125.5(\mathrm{ArCH}), 127.7(2 \mathrm{xArCH}), 127.8(\mathrm{ArCH}), 128.8$ $(2 \times \mathrm{ArCH}), 128.9(2 \mathrm{xArCH}), 137.4\left(C_{\mathrm{ipso}}\right), 138.7\left(C_{\mathrm{ipso}}\right), 156.5(\mathrm{OC}=\mathrm{ON}), 168.4\left(\mathrm{NC}=\mathrm{OCH}_{2}\right)$, $168.7\left(\mathrm{C}=\mathrm{ONCH}_{2} \mathrm{Ph}\right)$. MS (IE) m/z (\%): $395\left(\mathrm{M}^{+}, 1\right), 295$ (57), 189 (6), 161 (100), 105 (10), 91 (13), 57 (58), 41 (6). IR $v_{\max }(\mathrm{KBr}) \mathrm{cm}^{-1}:$ 3419, 3280, 1721, 1708, 1678, 1534, 1317, 1159, 746.

(S)-tert-Butyl 3-oxo-2-phenyl-5-[(S)-1-phenylethylcarbamoyl]pyrazolidine-1-carbo-xylate, $(\boldsymbol{S}, \boldsymbol{S}-10)$. The General Procedure was followed with $0.55 \mathrm{~g}(1.79 \mathrm{mmol})$ of $(S)-7,0.19 \mathrm{~g}$ $(1.88 \mathrm{mmol}, 0.27 \mathrm{~mL})$ of $\mathrm{Et}_{3} \mathrm{~N}, 0.23 \mathrm{~g}(1.88 \mathrm{mmol}, 0.23 \mathrm{~mL})$ of pivaloyl chloride, $0.23 \mathrm{~g}$ (1.88 mmol, $0.24 \mathrm{~mL}$ ) of $(S)$ - $\alpha$-methylbenzylamine $(S)-5$ and $35 \mathrm{~mL}$ of THF to obtain the $N$-Boc protected amide $(S, S)-10(0.51 \mathrm{~g}, 69 \%$ yield $)$ as a white solid, mp $164-166^{\circ} \mathrm{C},[\alpha]_{\mathrm{D}}{ }^{20}=-93.1(\mathrm{c}$ 1, MeOH). ${ }^{1} \mathrm{H}-\mathrm{NMR}\left(\mathrm{CDCl}_{3}, 400 \mathrm{MHz}\right): \delta=1.31\left(\mathrm{~s}, 9 \mathrm{H}, \mathrm{C}\left(\mathrm{CH}_{3}\right)_{3}\right), 1.44(\mathrm{~d}, J=6.8 \mathrm{~Hz}, 3 \mathrm{H}$, $\left.{ }^{*} \mathrm{CHCH}_{3}\right), 3.13\left(\mathrm{dd}, J=9.6,17.3 \mathrm{~Hz}, 1 \mathrm{H}, \mathrm{CH}_{2}\right), 3.25$ (dd, $\left.J=1.8,17.4 \mathrm{~Hz}, 1 \mathrm{H}, \mathrm{CH}_{2}\right), 4.98(\mathrm{dd}, J$ $\left.=1.8,9.5 \mathrm{~Hz}, 1 \mathrm{H},{ }^{*} \mathrm{CH}\right), 5.09\left(\mathrm{dq}, J=7.0,7.3 \mathrm{~Hz}, 1 \mathrm{H}, \mathrm{NH}^{*} \mathrm{CHCH}_{3}\right), 6.96(\mathrm{~d}, J=7.7 \mathrm{~Hz}, 1 \mathrm{H}$, $\mathrm{NH}), 7.17-7.43(\mathrm{~m}, 8 \mathrm{H}, \mathrm{ArCH}), 7.50-7.56(\mathrm{~m}, 2 \mathrm{H}, \mathrm{ArCH}) .{ }^{13} \mathrm{C}-\mathrm{NMR}\left(\mathrm{CDCl}_{3}, 100.5 \mathrm{MHz}\right): \delta=$ $22.0\left({ }^{*} \mathrm{CHCH}_{3}\right), 27.8\left(\mathrm{C}\left(\mathrm{CH}_{3}\right)_{3}\right), 34.4\left(\mathrm{CH}_{2}\right), 49.5\left({ }^{*} \mathrm{CHCH}_{3}\right), 59.8\left({ }^{*} \mathrm{CH}\right), 84.9\left(\mathrm{C}_{\left.\left(\mathrm{CH}_{3}\right)_{3}\right), 119.3}\right.$ (2xArCH), $125.6(\mathrm{ArCH}), 126.2(2 \mathrm{xArCH}), 127.8(\mathrm{ArCH}), 128.9(2 \mathrm{xArCH}), 129.0(2 \mathrm{xArCH})$, $138.8\left(C_{\mathrm{ipso}}\right), 142.6\left(C_{\mathrm{ipso}}\right), 156.5(\mathrm{OC}=\mathrm{ON}), 167.9\left(\mathrm{NC}=\mathrm{OCH}_{2}\right), 168.6\left({ }^{*} \mathrm{CHC}=\mathrm{ON}\right) . \mathrm{MS}(\mathrm{IE})$ 
m/z (\%): 409 ( $\left.\mathrm{M}^{+}, 1\right), 309$ (52), 205 (35), 161 (100), 119 (4), 105 (88), 77 (6), 57 (70), 41 (6). IR $v_{\max }(\mathrm{KBr}) \mathrm{cm}^{-1}: 3339,2919,1739,1720,1675,1532,1306,1153,756$. HRMS (ESI-TOF) calculated for $\mathrm{C}_{23} \mathrm{H}_{27} \mathrm{~N}_{3} \mathrm{O}_{4}+\mathrm{H}^{+}$: 410.2074; Found: 410.2074.

(S)-tert-Butyl 5-[(S)-1-hydroxybutan-2-ylcarbamoyl]-3-oxo-2-phenylpyrazolidine-1carboxylate, (S,S-11). The General Procedure was followed with $0.55 \mathrm{~g}(1.79 \mathrm{mmol})$ of $(S)-7$, $0.19 \mathrm{~g}(1.88 \mathrm{mmol}, 0.27 \mathrm{~mL})$ of $\mathrm{Et}_{3} \mathrm{~N}, 0.23 \mathrm{~g}(1.88 \mathrm{mmol}, 0.23 \mathrm{~mL})$ of pivaloyl chloride, $0.17 \mathrm{~g}$ (1.88 mmol, $0.18 \mathrm{~mL}$ ) of (S)-2-aminobutan-1-ol and $35 \mathrm{~mL}$ of THF to obtain the $N$-Boc protected amide $(S, S)-11(0.52 \mathrm{~g}, 77 \%$ yield $)$ as a white solid, mp $174-176{ }^{\circ} \mathrm{C},[\alpha]_{\mathrm{D}}{ }^{20}=-31.0(\mathrm{c}$ 1, MeOH). ${ }^{1} \mathrm{H}-\mathrm{NMR}\left(\mathrm{CDCl}_{3}, 400 \mathrm{MHz}\right): \delta=0.79\left(\mathrm{t}, J=7.5 \mathrm{~Hz}, 3 \mathrm{H}, \mathrm{CH}_{2} \mathrm{CH}_{3}\right), 1.30(\mathrm{~s}, 9 \mathrm{H}$, $\left.\mathrm{C}\left(\mathrm{CH}_{3}\right)_{3}\right), 1.36-1.47\left(\mathrm{~m}, 1 \mathrm{H}, \mathrm{CH}_{2} \mathrm{CH}_{3}\right), 1.51-1.63\left(\mathrm{~m}, 1 \mathrm{H}, \mathrm{CH}_{2} \mathrm{CH}_{3}\right), 2.48(\mathrm{br}, 1 \mathrm{H}, \mathrm{OH}), 3.15(\mathrm{dd}$, $\left.J=9.2,17.2 \mathrm{~Hz}, 1 \mathrm{H}, \mathrm{CH}_{2}\right), 3.24\left(\mathrm{dd}, J=2.0,17.4 \mathrm{~Hz}, 1 \mathrm{H}, \mathrm{CH}_{2}\right), 3.61-3.68(\mathrm{~m}, 1 \mathrm{H}$, $\left.{ }^{*} \mathrm{CHCH}_{2} \mathrm{OH}\right), 3.69-3.77\left(\mathrm{~m}, 1 \mathrm{H},{ }^{*} \mathrm{CHCH}_{2} \mathrm{OH}\right), 3.82-3.92\left(\mathrm{~m}, 1 \mathrm{H},{ }^{*} \mathrm{CHCH}_{2} \mathrm{OH}\right), 5.03(\mathrm{dd}, J=$ 2.0, 9.3 Hz, $\left.1 \mathrm{H},{ }^{*} \mathrm{CH}\right), 6.86(\mathrm{~d}, J=8.0 \mathrm{~Hz}, 1 \mathrm{H}, \mathrm{NH}), 7.14-7.20(\mathrm{~m}, 1 \mathrm{H}, \operatorname{ArCH}), 7.33-7.40(\mathrm{~m}$, $2 \mathrm{H}, \mathrm{ArCH}), 7.49-7.56(\mathrm{~m}, 2 \mathrm{H}, \mathrm{ArCH}) .{ }^{13} \mathrm{C}-\mathrm{NMR}\left(\mathrm{CDCl}_{3}, 100.5 \mathrm{MHz}\right): \delta=10.4\left(\mathrm{CH}_{2} \mathrm{CH}_{3}\right), 24.2$

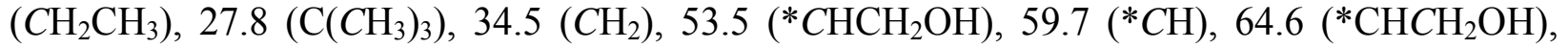

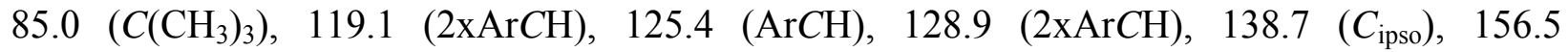
$(\mathrm{OC}=\mathrm{ON}), 168.4\left(\mathrm{NC}=\mathrm{OCH}_{2}\right), 169.3\left({ }^{*} \mathrm{CHC}=\mathrm{ON}\right) . \mathrm{MS}(\mathrm{IE}) \mathrm{m} / \mathrm{z}(\%): 377\left(\mathrm{M}^{+}, 1\right), 277(41), 218$ (3), 171 (17), 161 (100), 108 (8), 77 (3), 57 (68), 41 (5). IR $v_{\max }(\mathrm{KBr}) \mathrm{cm}^{-1}: 3442,3298,2980$, $1720,1698,1655,1543,1314,1160,769$.

\section{General procedure for the $\mathbf{N}$-tert-butoxycarbonyl group removal}

The required $\mathrm{N}$-Boc-amide was dissolved in $\mathrm{CH}_{2} \mathrm{Cl}_{2}$ and 18 equiv. of trifluoroacetic acid was added. The resulting reaction mixture was stirred during $2 \mathrm{~h}$ at room temperature and then the mixture was extracted five times with a saturated solution of $\mathrm{NaHCO}_{3}$. The aqueous phase was extracted twice with $\mathrm{CH}_{2} \mathrm{Cl}_{2}$ and the organic phase was washed with brine, dried with anhyd. $\mathrm{Na}_{2} \mathrm{SO}_{4}$ and concentrated in the rotary evaporator. The corresponding product was recrystallised from a mixture of hexane-EtOAc $(95: 5 \mathrm{v} / \mathrm{v})$ or purified by column chromatography. The products were dried at $40{ }^{\circ} \mathrm{C}$ under vacuum.

(R)-5-Oxo- $N$, 1-diphenylpyrazolidine-3-carboxamide, $(\boldsymbol{R}-12)$. The General Procedure was followed with $0.54 \mathrm{~g}(1.41 \mathrm{mmol})$ of $(R)-\mathbf{8}$ to obtain amide $(R)-\mathbf{1 2}$. This product was recrystallised from hexane-EtOAc $(95: 5 \mathrm{v} / \mathrm{v})$ to give $0.38 \mathrm{~g}$ (96\% yield) of $(R)-\mathbf{1 2}$ as a white solid, mp 190-192 ${ }^{\circ} \mathrm{C},[\alpha]_{\mathrm{D}}{ }^{20}=+136.5\left(c 1, \mathrm{CHCl}_{3}\right) .{ }^{1} \mathrm{H}-\mathrm{NMR}\left(\mathrm{CDCl}_{3}, 400 \mathrm{MHz}\right): \delta=3.10(\mathrm{dd}$, $J=9.0,17.0 \mathrm{~Hz}, 1 \mathrm{H}, \mathrm{CH}_{2}$ ), 3.18 (dd, $J=4.2,17.0 \mathrm{~Hz}, 1 \mathrm{H}, \mathrm{CH}_{2}$ ), 4.23 (ddd, $J=4.4,6.4,8.8 \mathrm{~Hz}$, $\left.1 \mathrm{H},{ }^{*} \mathrm{CH}\right), 6.00(\mathrm{~d}, J=5.8 \mathrm{~Hz}, 1 \mathrm{H}, \mathrm{PhNNH}), 7.04-7.15(\mathrm{~m}, 2 \mathrm{H}, \mathrm{ArCH}), 7.23-7.37(\mathrm{~m}, 4 \mathrm{H}$, $\mathrm{ArCH})$, 7.44-7.48 (m, $2 \mathrm{H}, \operatorname{ArCH}), 7.82-7.87(\mathrm{~m}, 2 \mathrm{H}, \mathrm{ArCH}), 9.32(\mathrm{br}, 1 \mathrm{H}, \mathrm{NH}) .{ }^{13} \mathrm{C}-\mathrm{NMR}$ $\left(\mathrm{CDCl}_{3}, 100.5 \mathrm{MHz}\right): \delta=37.5\left(\mathrm{CH}_{2}\right), 56.0\left({ }^{*} \mathrm{CH}\right), 117.9(2 \mathrm{xArCH}), 119.8(2 \mathrm{xArCH}), 124.60$ $(\mathrm{ArCH}), 124.63(\mathrm{ArCH}), 128.9(2 \times \mathrm{xArCH}), 129.0(2 \times \mathrm{xACH}), 137.3\left(C_{\mathrm{ipso}}\right), 138.6\left(C_{\mathrm{ipso}}\right), 168.7$ $\left(\mathrm{NC}=\mathrm{OCH}_{2}\right), 169.6(\mathrm{C}=\mathrm{ONPh})$. MS (IE) $\mathrm{m} / \mathrm{z}(\%): 282\left(\mathrm{M}^{+}+1,9\right), 281\left(\mathrm{M}^{+}, 100\right), 280\left(\mathrm{M}^{+}-1\right.$, 24), 206 (8), 161 (93), 93 (52), 66 (3). IR $v_{\max }(\mathrm{KBr}) \mathrm{cm}^{-1}:$ 3446, 3313, 2421, 1708, 1687, 1531, 
1360, 1065, 756. Anal. Calcd. for $\mathrm{C}_{16} \mathrm{H}_{15} \mathrm{~N}_{3} \mathrm{O}_{2}$ (281.31): C 68.31, H 5.37, N 14.94; Found: C 68.61, H 5.71, N 15.03\%.

(R)- $N$-Benzyl-5-oxo-1-phenylpyrazolidine-3-carboxamide, $(\boldsymbol{R}-13)$. The General Procedure was followed with $0.43 \mathrm{~g}(1.09 \mathrm{mmol})$ of $(R)-9$ to obtain crude amide $(R)-13$, which was recrystallised from hexane-EtOAc $(95: 5 \mathrm{v} / \mathrm{v})$ to afford $0.27 \mathrm{~g}(85 \%$ yield $)$ of $(R)-13$ as a white solid, mp $158-160{ }^{\circ} \mathrm{C},[\alpha]_{\mathrm{D}}{ }^{20}=+86.1\left(\mathrm{c} 1, \mathrm{CHCl}_{3}\right) .{ }^{1} \mathrm{H}-\mathrm{NMR}\left(\mathrm{CDCl}_{3}, 400 \mathrm{MHz}\right): \delta=3.14(\mathrm{dd}$, $\left.J=9.2,17.2 \mathrm{~Hz}, 1 \mathrm{H}, \mathrm{CH}_{2}\right), 3.26\left(\mathrm{dd}, J=2.9,17.2 \mathrm{~Hz}, 1 \mathrm{H}, \mathrm{CH}_{2}\right), 4.20$ (ddd, $J=2.9,6.2,9.5 \mathrm{~Hz}$, $1 \mathrm{H}, * \mathrm{CH}), 4.41\left(\mathrm{dd}, J=5.7,14.8 \mathrm{~Hz}, 1 \mathrm{H}, \mathrm{CH}_{2} \mathrm{Ph}\right), 4.50(\mathrm{dd}, 1 \mathrm{H}, J=5.8,15.0 \mathrm{~Hz}), 5.33(\mathrm{~d}, J=$ 6.6 Hz, 1H, PhNNH), 7.13-7.19 (m, 3H, ArCH), 7.25-7.29 (m, 3H, ArCH), 7.33-7.39 (m, 2H, $\mathrm{ArCH}), 7.48$ (br, $\left.1 \mathrm{H}, \mathrm{NHCH}_{2} \mathrm{Ph}\right), 7.76-7.80$ (m, $\left.2 \mathrm{H}, \mathrm{ArH}\right) .{ }^{13} \mathrm{C}-\mathrm{NMR}\left(\mathrm{CDCl}_{3}, 100.5 \mathrm{MHz}\right): \delta=$ $37.6\left(\mathrm{CH}_{2}\right), 43.4\left(\mathrm{CH}_{2} \mathrm{Ph}\right), 55.7\left({ }^{*} \mathrm{CH}\right), 118.1(2 \mathrm{xArCH}), 124.6(\mathrm{ArCH}), 127.4(2 \times \mathrm{ArCH}), 127.5$ $(\mathrm{ArCH}), 128.7(2 \mathrm{xArCH}), 128.9(2 \mathrm{xArCH}), 137.8\left(C_{\mathrm{ipso}}\right), 138.6\left(C_{\mathrm{ipso}}\right), 169.8\left(\mathrm{NC}=\mathrm{OCH}_{2}\right)$, $170.3\left(\mathrm{C}=\mathrm{ONCH}_{2} \mathrm{Ph}\right)$. MS (IE) m/z (\%): $296\left(\mathrm{M}^{+}+1,9\right), 295\left(\mathrm{M}^{+}, 49\right), 189$ (7), 161 (100), 108 (6), 91 (6). IR $v_{\max }(\mathrm{KBr}) \mathrm{cm}^{-1}: 3448,3390,3210,2171,1684,1594,1361,1007,762$.

(R)-5-Oxo-1-phenyl- $N$ - $[(S)$-1-phenylethyl]pyrazolidine-3-carboxamide, $\quad(R, S-14) . \quad$ The General Procedure was followed with $0.55 \mathrm{~g}(1.34 \mathrm{mmol})$ of $(R, S)-10$ to obtain crude amide $(R, S)-14$, which was recrystallised from hexane-EtOAc $(95: 5 \mathrm{v} / \mathrm{v})$ to give $0.41 \mathrm{~g}(99 \%$ yield) of $(R, S)-14$ as a white solid, mp $118-120{ }^{\circ} \mathrm{C},[\alpha]_{\mathrm{D}}{ }^{20}=+73.1\left(\mathrm{c} 1, \mathrm{CHCl}_{3}\right) .{ }^{1} \mathrm{H}-\mathrm{NMR}\left(\mathrm{CDCl}_{3}, 400\right.$ $\mathrm{MHz}): \delta=1.45\left(\mathrm{~d}, J=6.6 \mathrm{~Hz}, 3 \mathrm{H},{ }^{*} \mathrm{CHCH}_{3}\right), 2.99\left(\mathrm{dd}, J=8.6,17.4 \mathrm{~Hz}, 1 \mathrm{H}, \mathrm{CH}_{2}\right), 3.10(\mathrm{dd}, J=$ 3.1, $\left.17.0 \mathrm{~Hz}, 1 \mathrm{H}, \mathrm{CH}_{2}\right), 4.04-4.14\left(\mathrm{~m}, 1 \mathrm{H},{ }^{*} \mathrm{CH}\right), 5.04\left(\mathrm{dq}, 1 \mathrm{H}, J=7.3,7.3 \mathrm{~Hz},{ }^{*} \mathrm{CHCH}_{3}\right), 5.54$ (br, $1 \mathrm{H}, \mathrm{PhNNH}), 7.02-7.07$ (m, 2H, ArCH), 7.11-7.19 (m, 4H, ArCH), 7.30-7.37 (m, 2H, $\mathrm{ArCH}), 7.58$ (br, 1H, $\left.\mathrm{NH}^{*} \mathrm{CHCH}_{3}\right), 7.69-7.75$ (m, 2H, $\left.\mathrm{ArCH}\right) .{ }^{13} \mathrm{C}-\mathrm{NMR}\left(\mathrm{CDCl}_{3}, 100.5 \mathrm{MHz}\right)$ : $\delta=22.2\left({ }^{*} \mathrm{CHCH}_{3}\right), 37.4\left(\mathrm{CH}_{2}\right), 48.9\left({ }^{*} \mathrm{CHCH}_{3}\right), 56.0\left({ }^{*} \mathrm{CH}\right), 118.1(2 \times \mathrm{ArCH}), 124.9(\mathrm{ArCH})$, $125.6(2 \mathrm{xArCH}), 127.4(\mathrm{ArCH}), 128.7(2 \mathrm{xArCH}), 129.0(2 \mathrm{xArCH}), 138.5\left(C_{\mathrm{ipso}}\right), 142.6\left(C_{\mathrm{ipso}}\right)$, $169.1\left(\mathrm{NC}=\mathrm{OCH}_{2}\right), 169.8\left({ }^{*} \mathrm{CHC}=\mathrm{ON}\right) . \mathrm{MS}(\mathrm{IE}) \mathrm{m} / \mathrm{z}(\%): 310\left(\mathrm{M}^{+}+1,18\right), 309\left(\mathrm{M}^{+}, 83\right), 205$ (100), 161 (98), 105 (81) 77 (7). IR $v_{\max }(\mathrm{KBr}) \mathrm{cm}^{-1}:$ 3313, 3238, 1952, 1687, 1657, 1528, 1360, 755, 699. HRMS (ESI-TOF) calculated for $\mathrm{C}_{18} \mathrm{H}_{19} \mathrm{~N}_{3} \mathrm{O}_{2}+\mathrm{H}^{+}: 310.1550$; Found: 310.1553 .

(R)- $N$-[(S)-1-Hydroxybutan-2-yl]-5-oxo-1-phenylpyrazolidine-3-carboxamide, $(R, S-15)$. The General Procedure was followed with $0.49 \mathrm{~g}(1.3 \mathrm{mmol})$ of $(R, S)-11$ to obtain crude amide $(R, S)$ 15, which was purified by column chromatography using a mixture of EtOAc-hexane $(8: 2 \mathrm{v} / \mathrm{v})$ as eluent to give $0.26 \mathrm{~g}(72 \%$ yield $)$ of $(R, S)-15$ as a white solid, mp 106-108 ${ }^{\circ} \mathrm{C},[\alpha]_{\mathrm{D}}{ }^{20}=+53.0(\mathrm{C}$ 1, MeOH). ${ }^{1} \mathrm{H}-\mathrm{NMR}\left(\mathrm{CDCl}_{3}, 300 \mathrm{MHz}\right): \delta=0.91\left(\mathrm{t}, J=7.4 \mathrm{~Hz}, 3 \mathrm{H}, \mathrm{CH}_{2} \mathrm{CH}_{3}\right), 1.43-1.67(\mathrm{~m}$, $2 \mathrm{H}, \mathrm{CH}_{2} \mathrm{CH}_{3}$ ), 2.50 (br, $\left.1 \mathrm{H}, \mathrm{OH}\right), 3.04$ (dd, $\left.J=9.0,17.3 \mathrm{~Hz}, 1 \mathrm{H}, \mathrm{CH}_{2}\right), 3.15$ (dd, $J=4.7,17.2$ $\left.\mathrm{Hz}, 1 \mathrm{H}, \mathrm{CH}_{2}\right), 3.47-3.57\left(\mathrm{~m}, 2 \mathrm{H}, \mathrm{CH}_{2} \mathrm{OH}\right), 3.73-3.84\left(\mathrm{~m}, 1 \mathrm{H},{ }^{*} \mathrm{CHCH}_{2} \mathrm{OH}\right), 4.06-4.16(\mathrm{~m}, 1 \mathrm{H}$, $\left.{ }^{*} \mathrm{CH}\right), 5.50(\mathrm{~d}, J=6.9 \mathrm{~Hz}, 1 \mathrm{H}, \mathrm{PhNNH}), 7.12-7.17(\mathrm{~m}, 1 \mathrm{H}, \mathrm{ArCH}), 7.33-7.39(\mathrm{~m}, 3 \mathrm{H}, \mathrm{C}=\mathrm{ONH}$, $\mathrm{ArCH}), 7.77-7.84(\mathrm{~m}, 2 \mathrm{H}, \mathrm{ArCH}) .{ }^{13} \mathrm{C}-\mathrm{NMR}\left(\mathrm{CDCl}_{3}, 75.5 \mathrm{MHz}\right): \delta=10.6\left(\mathrm{CH}_{2} \mathrm{CH}_{3}\right), 24.2$ $\left(\mathrm{CH}_{2} \mathrm{CH}_{3}\right), 37.6\left(\mathrm{CH}_{2}\right), 53.3\left({ }^{*} \mathrm{CHCH}_{2} \mathrm{OH}\right), 55.8\left({ }^{*} \mathrm{CH}\right), 64.4\left({ }^{*} \mathrm{CHCH}_{2} \mathrm{OH}\right), 118.4(2 \times \mathrm{ArCH})$, $125.1(\mathrm{ArCH}), 129.2(2 \times \mathrm{ArCH}), 138.3\left(C_{\mathrm{ipso}}\right), 169.8\left(\mathrm{NC}=\mathrm{OCH}_{2}\right), 170.6\left({ }^{*} \mathrm{CHC}=\mathrm{ON}\right) . \mathrm{MS}(\mathrm{IE})$ m/z (\%): $278\left(\mathrm{M}^{+}+1,3\right), 277\left(\mathrm{M}^{+}, 15\right), 171$ (23), 161 (100), 153 (11), 108 (14), 58 (14), 43 (5). 
IR $v_{\max }(\mathrm{KBr}) \mathrm{cm}^{-1}: 3328,3244,2876,1693,1646,1548,1497,1362,1220,756$. HRMS (ESITOF) calculated for $\mathrm{C}_{14} \mathrm{H}_{10} \mathrm{~N}_{3} \mathrm{O}_{3}+\mathrm{H}^{+}: 278.1499$; Found: 278.1498 .

(S)-5-Oxo- $N$-1-diphenylpyrazolidine-3-carboxamide, (S-12). The General Procedure was followed with $0.56 \mathrm{~g}(1.46 \mathrm{mmol})$ of $(S)-8$ to obtain crude amide $(S)-12$, which was recrystallised from hexane-EtOAc $(95: 5 \mathrm{v} / \mathrm{v})$ to afford $0.38 \mathrm{~g}(92 \%$ yield) of $(S)$-12 as a white solid, mp 188$190{ }^{\circ} \mathrm{C},[\alpha]_{\mathrm{D}}{ }^{20}=-132.7\left(c 1, \mathrm{CHCl}_{3}\right) .{ }^{1} \mathrm{H}-\mathrm{NMR}\left(\mathrm{CDCl}_{3}, 400 \mathrm{MHz}\right): \delta=3.21(\mathrm{dd}, J=9.7,17.6$ $\left.\mathrm{Hz}, 1 \mathrm{H}, \mathrm{CH}_{2}\right), 3.29$ (dd, $\left.J=2.9,17.6 \mathrm{~Hz}, 1 \mathrm{H}, \mathrm{CH}_{2}\right), 4.28\left(\mathrm{~d}, J=8.8 \mathrm{~Hz}, 1 \mathrm{H},{ }^{*} \mathrm{CH}\right), 5.48(\mathrm{br}, 1 \mathrm{H}$, $\mathrm{PhNNH}$ ), 7.10-7.22 (m, 2H, ArCH), 7.29-7.35 (m, 2H, ArCH), 7.39-7.53 (m, 4H, ArCH), 7.867.91 (m, 2H, $\mathrm{ArCH}), 9.11$ (br, $1 \mathrm{H}, \mathrm{NHPh}) .{ }^{13} \mathrm{C}-\mathrm{NMR}\left(\mathrm{CDCl}_{3}, 100.5 \mathrm{MHz}\right): \delta=37.4\left(\mathrm{CH}_{2}\right), 55.9$ $\left({ }^{*} \mathrm{CH}\right), 117.9(2 \mathrm{xArCH}), 119.8(2 \mathrm{xArCH}), 124.5(\mathrm{ArCH}), 124.6(\mathrm{ArCH}), 128.9(2 \mathrm{xArCH}), 129.0$ $(2 \times A r C H), 137.3\left(C_{\text {ipso }}\right), 138.6\left(C_{\text {ipso }}\right), 168.7\left(\mathrm{NC}=\mathrm{OCH}_{2}\right), 169.6(\mathrm{C}=\mathrm{ONPh}) . \mathrm{MS}(\mathrm{IE}) \mathrm{m} / \mathrm{z}(\%)$ : $282\left(\mathrm{M}^{+}+1,19\right), 281\left(\mathrm{M}^{+}, 83\right), 175$ (16), 161 (100), 134 (11), 93 (19), 77 (6), 65 (3). IR $v_{\max }$ (KBr) $\mathrm{cm}^{-1}:$ 3446, 3313, 2345, 1708, 1687, 1531, 1360, 1065, 756.

(S)-N-Benzyl-5-oxo-1-phenylpyrazolidine-3-carboxamide, (S-13). The General Procedure was followed with $0.53 \mathrm{~g}(1.34 \mathrm{mmol})$ of $(S)-\mathbf{9}$ to obtain crude amide $(S)-\mathbf{1 3}$, which was recrystallised from hexane-EtOAc $(95: 5 \mathrm{v} / \mathrm{v})$ to give $0.37 \mathrm{~g}$ (94 \% yield) of $(S)$-13 as a white solid, mp 156$158^{\circ} \mathrm{C},[\alpha]_{\mathrm{D}}{ }^{20}=-85.6\left(\mathrm{c} 1, \mathrm{CHCl}_{3}\right) .{ }^{1} \mathrm{H}-\mathrm{NMR}\left(\mathrm{CDCl}_{3}, 400 \mathrm{MHz}\right): \delta=3.11(\mathrm{dd}, J=9.5,17.2$ $\left.\mathrm{Hz}, 1 \mathrm{H}, \mathrm{CH}_{2}\right), 3.23\left(\mathrm{dd}, J=3.3,17.2 \mathrm{~Hz}, 1 \mathrm{H}, \mathrm{CH}_{2}\right), 4.17$ (ddd, $J=3.1,6.4,9.5 \mathrm{~Hz}, 1 \mathrm{H},{ }^{*} \mathrm{CH}$ ), $4.40\left(\mathrm{dd}, J=5.9,15.0 \mathrm{~Hz}, 1 \mathrm{H}, \mathrm{CH}_{2} \mathrm{Ph}\right), 4.49$ (dd, $\left.J=5.9,15.0 \mathrm{~Hz}, 1 \mathrm{H}, \mathrm{CH}_{2} \mathrm{Ph}\right), 5.36$ (d, $J=6.6$ $\mathrm{Hz}, 1 \mathrm{H}, \mathrm{PhNNH}), 7.12-7.18(\mathrm{~m}, 3 \mathrm{H}, \mathrm{ArCH}), 7.24-7.28(\mathrm{~m}, 3 \mathrm{H}, \mathrm{ArCH}), 7.32-7.38(\mathrm{~m}, 2 \mathrm{H}$, $\mathrm{ArCH}), 7.48$ (br, $\left.1 \mathrm{H}, \mathrm{NHCH}_{2} \mathrm{Ph}\right), 7.75-7.80(\mathrm{~m}, 2 \mathrm{H}, \mathrm{ArCH}) .{ }^{13} \mathrm{C}-\mathrm{NMR}\left(\mathrm{CDCl}_{3}, 100.5 \mathrm{MHz}\right): \delta$ $=37.5\left(\mathrm{CH}_{2}\right), 43.4\left(\mathrm{CH}_{2} \mathrm{Ph}\right), 55.7\left({ }^{*} \mathrm{CH}\right), 118.1(2 \times \mathrm{ArCH}), 124.6(\mathrm{ArCH}), 127.4(2 \times \mathrm{ArCH})$, $127.5(\mathrm{ArCH}), 128.7(2 \times \mathrm{xArCH}), 128.9(2 \mathrm{xArCH}), 137.7\left(C_{\mathrm{ipso}}\right), 138.5\left(C_{\text {ipso }}\right), 169.7$ $\left(\mathrm{NC}=\mathrm{OCH}_{2}\right), 170.2\left(C=\mathrm{ONCH}_{2} \mathrm{Ph}\right)$. MS (IE) $\mathrm{m} / \mathrm{z}(\%): 296\left(\mathrm{M}^{+}+1,20\right), 295\left(\mathrm{M}^{+}, 100\right), 253(4)$, 189 (5), 161 (93), 91 (10), 77 (6). IR $v_{\max }(\mathrm{KBr}) \mathrm{cm}^{-1}: 3446,3398,3210,2331,1684,1594$, 1361, 1007, 762. Anal. Calcd. for $\mathrm{C}_{17} \mathrm{H}_{17} \mathrm{~N}_{3} \mathrm{O}_{2}$ (295.34): C 69.14, H 5.80, N 14.23; Found: $\mathrm{C}$ 69.46, H 6.00, N 14.44\%.

(S)-5-Oxo-1-phenyl- $N$ - $[(S)$-1-phenylethyl]pyrazolidine-3-carboxamide, $\quad(S, S-14) . \quad$ The General Procedure was followed with $0.475 \mathrm{~g}(1.16 \mathrm{mmol})$ of $(S, S)-\mathbf{1 0}$ to obtain crude amide $(S, S)-14$, which was recrystallised from hexane-EtOAc $(95: 5 \mathrm{v} / \mathrm{v})$ to give $0.36 \mathrm{~g}$ (99\% yield) of $(S, S)-14$ as a white solid, mp $152-154{ }^{\circ} \mathrm{C},[\alpha]_{\mathrm{D}}{ }^{20}=-83.6\left(\right.$ c $\left.1, \mathrm{CHCl}_{3}\right) .{ }^{1} \mathrm{H}-\mathrm{NMR}\left(\mathrm{CDCl}_{3}, 400\right.$ MHz): $\delta=1.36\left(\mathrm{~d}, J=7.0 \mathrm{~Hz}, 3 \mathrm{H},{ }^{*} \mathrm{CHCH}_{3}\right), 3.00\left(\mathrm{dd}, J=9.5,17.2 \mathrm{~Hz}, 1 \mathrm{H}, \mathrm{CH}_{2}\right), 3.13(\mathrm{dd}, J=$ 2.9, $\left.17.2 \mathrm{~Hz}, 1 \mathrm{H}, \mathrm{CH}_{2}\right), 4.04$ (ddd, $\left.J=3.1,6.0,9.3 \mathrm{~Hz}, 1 \mathrm{H},{ }^{*} \mathrm{CH}\right), 5.02(\mathrm{dq}, J=7.0,7.2 \mathrm{~Hz}, 1 \mathrm{H}$, $\left.{ }^{*} \mathrm{CHCH}_{3}\right), 5.40(\mathrm{~d}, J=6.2 \mathrm{~Hz}, 1 \mathrm{H}, \mathrm{PhNNH}), 7.14-7.20(\mathrm{~m}, 1 \mathrm{H}, \mathrm{ArCH}), 7.24-7.42(\mathrm{~m}, 7 \mathrm{H}$, $\mathrm{ArCH}), 7.46$ (br, $\left.1 \mathrm{H}, \mathrm{NH}^{*} \mathrm{CHCH}_{3}\right), 7.78-7.85(\mathrm{~m}, 1 \mathrm{H}, \mathrm{ArCH}) .{ }^{13} \mathrm{C}-\mathrm{NMR}\left(\mathrm{CDCl}_{3}, 100.5 \mathrm{MHz}\right)$ : $\delta=22.0\left({ }^{*} \mathrm{CHCH}_{3}\right), 37.4\left(\mathrm{CH}_{2}\right), 49.2\left({ }^{*} \mathrm{CHCH}_{3}\right), 55.8\left({ }^{*} \mathrm{CH}\right), 118.0(2 \mathrm{xArCH}), 124.9(\mathrm{ArCH})$, $126.1(2 \times \mathrm{ArCH}), 127.7(\mathrm{ArCH}), 128.9(2 \times \mathrm{xArCH}), 129.1(2 \times \mathrm{ArCH}), 138.6\left(C_{\mathrm{ipso}}\right), 142.9\left(C_{\mathrm{ipso}}\right)$, $169.1\left(\mathrm{NC}=\mathrm{OCH}_{2}\right), 169.7\left({ }^{*} \mathrm{CHC}=\mathrm{ON}\right) . \mathrm{MS}(\mathrm{IE}) \mathrm{m} / \mathrm{z}(\%): 309\left(\mathrm{M}^{+}, 43\right), 282(5), 205(73), 161$ (100), 105 (95), 77 (7). IR $v_{\max }(\mathrm{KBr}) \mathrm{cm}^{-1}:$ 3446, 3314, 3235, 2358, 1963, 1691, 1652, 1550, 
1365, 760. Anal. Calcd. for $\mathrm{C}_{18} \mathrm{H}_{19} \mathrm{~N}_{3} \mathrm{O}_{2}$ (309.36): C 69.88, H 6.19, N 13.58; Found: C 69.75, $\mathrm{H}$ $6.46, \mathrm{~N} 13.90 \%$.

(S)- $N$-[(S)-1-Hydroxybutan-2-yl]-5-oxo-1-phenylpyrazolidine-3-carboxamide, $(S, S-15)$. The General Procedure was followed with $0.46 \mathrm{~g}(1.22 \mathrm{mmol})$ of $(S, S)-11$ to obtain crude amide $(S, S)-15$, which was purified by gradient column chromatography [EtOAc $\rightarrow$ EtOAc-MeOH (8:2 $v / v)]$ to give $0.38 \mathrm{~g}(81 \%$ yield $)$ of $(S, S)-15$ as a white solid, mp $184-186{ }^{\circ} \mathrm{C},[\alpha]_{\mathrm{D}}{ }^{20}=-100.0(\mathrm{c}$ 1, MeOH). ${ }^{1} \mathrm{H}-\mathrm{NMR}\left(\mathrm{CDCl}_{3}+\mathrm{DMSO}_{6} 5: 1 \mathrm{v} / \mathrm{v}, 400 \mathrm{MHz}\right) \delta: 0.63(\mathrm{t}, J=7.5 \mathrm{~Hz}, 3 \mathrm{H}$, $\mathrm{CH}_{2} \mathrm{CH}_{3}$ ), 1.18-1.27 (m, 1H, $\left.\mathrm{CH}_{2} \mathrm{CH}_{3}\right), 1.39-1.50\left(\mathrm{~m}, 1 \mathrm{H}, \mathrm{CH}_{2} \mathrm{CH}_{3}\right), 2.93$ (dd, J = 4.2, $16.7 \mathrm{~Hz}$, $\left.1 \mathrm{H}, \mathrm{CH}_{2}\right), 2.98\left(\mathrm{dd}, J=8.8,16.5 \mathrm{~Hz}, 1 \mathrm{H}, \mathrm{CH}_{2}\right), 3.42-3.46\left(\mathrm{~m}, 2 \mathrm{H}, \mathrm{CH}_{2} \mathrm{OH}\right), 3.64-3.72(\mathrm{~m}, 1 \mathrm{H}$, $\left.{ }^{*} \mathrm{CHCH}_{2} \mathrm{OH}\right), 4.05\left(\mathrm{dd}, J=4.2,8.6 \mathrm{~Hz}, 1 \mathrm{H},{ }^{*} \mathrm{CH}\right), 7.01-7.06(\mathrm{~m}, 1 \mathrm{H}, \mathrm{ArCH}), 7.24-7.29(\mathrm{~m}, 2 \mathrm{H}$, $\operatorname{ArCH}), 7.60(\mathrm{~d}, J=8.4 \mathrm{~Hz}, 1 \mathrm{H}, \mathrm{C}=\mathrm{ONH}), 7.75-7.80(\mathrm{~m}, 2 \mathrm{H}, \mathrm{ArCH}) .{ }^{13} \mathrm{C}-\mathrm{NMR}\left(\mathrm{CDCl}_{3}+\right.$ DMSO- $\left.d_{6} 5: 1 \quad v / v, 100.5 \mathrm{MHz}\right): \delta=10.3\left(\mathrm{CH}_{2} \mathrm{CH}_{3}\right), 24.2\left(\mathrm{CH}_{2} \mathrm{CH}_{3}\right), 37.7\left(\mathrm{CH}_{2}\right), 52.7$ $\left({ }^{*} \mathrm{CHCH}_{2} \mathrm{OH}\right), 55.7\left({ }^{*} \mathrm{CH}\right), 63.4\left({ }^{*} \mathrm{CHCH}_{2} \mathrm{OH}\right), 118.2(2 \times \mathrm{ArCH}), 124.3(\mathrm{ArCH}), 128.8$ (2xArCH), $139.0\left(C_{\text {ipso }}\right), 170.2\left(\mathrm{NC}=\mathrm{OCH}_{2}\right), 170.5\left({ }^{*} \mathrm{CHC}=\mathrm{ON}\right) . \mathrm{MS}(\mathrm{IE}) \mathrm{m} / \mathrm{z}(\%): 277\left(\mathrm{M}^{+}\right.$, 22), 218 (2), 171 (23), 161 (100), 119 (14), 108 (16), 58 (17), 43 (5). IR $v_{\max }(\mathrm{KBr}) \mathrm{cm}^{-1}: 3426$, $3275,3213,2961,2170,1688,1630,1361,1072$, 756. Anal. Calcd. for $\mathrm{C}_{14} \mathrm{H}_{19} \mathrm{~N}_{3} \mathrm{O}_{3}$ (277.32): C 60.63, H 6.91, N 15.15; Found: C 60.23, H 7.30, N 14.79\%.

\section{Acknowledgements}

The authors are grateful to Secretaría de Educación Pública (SEP, México) and Consejo Nacional de Ciencia y Tecnología (CONACyT, México) for financial support via grants No. 45157-Q, SEP-PADES No. 2009-01-09-006-220, and SEP-CONACyT No. 2006-60366. We are also thankful to Erika Jiménez-González and Marco A. Leyva-Ramírez for technical assistance.

\section{References and Notes}

1. (a) Panfil I.; Urbanczyk-Lipkowska, Z.; Suwinska, K., Solecka, J.; Chmielewski, M. Tetrahedron 2002, 58, 1199. (b) Küchenthal, C.-H.; Maison, W. Synthesis 2010, 719.

2. White, J. D.; Toske, S. G. Tetrahedron Lett. 1993, 34, 207.

3. Ege, S. N.; Tsui, E. Y.; Spencer, R. L.; Potter, B. E.; Eagleson, B. K.; Friedman, H. Z. J. Chem. Soc., Chem. Commun. 1974, 216.

4. Sibi, M. P.; Soeta, T. J. Am. Chem. Soc. 2007, 129, 4522.

5. Korotkov, V. S.; Larionov, O. V.; Hofmeister A.; Magull, J.; De Meijere, A., J. Org. Chem. 2007, 72, 7504.

6. (a) Melgar-Fernández, R.; Juaristi, E. Sixteenth International Conference on Organic Synthesis, Mérida, Yucatán, México, June 11-15, 2006: Abstract No. 240. (b) MelgarFernández, R.; Juaristi, E. J. Mex. Chem. Soc. 2006, 50, Special issue 1, 223. 
7. Tzeng, Z.-H.; Yang, S.-W.; Liu, P.-M.; Chen, K.-M. J. Chin. Chem. Soc. 2008, 55, 626.

8. For reviews on the use of $\alpha$-methylbenzylamine in asymmetric synthesis, see: (a) Juaristi, E.; Escalante, J.; León-Romo, J. L.; Reyes, A. Tetrahedron: Asymmetry 1998, 9, 715 . (b) Juaristi, E.; León-Romo, J. L.; Reyes, A. Tetrahedron: Asymmetry 1999, 10, 2441.

9. Bandala, Y.; Melgar-Fernández, R.; Guzmán-Mejía, R.; Olivares-Romero, J. L.; DíazSánchez, B. R.; González-Olvera, R.; Vargas-Caporali, J.; Juaristi, E. J. Mex. Chem. Soc. 2009, 53 (3), 147.

10. For a recent example, see: Ávila-Ortiz, C. G.; Reyes-Rangel, G.; Juaristi, E. Tetrahedron 2005, 61, 8372.

11. (a) Seebach, D.; Prelog, V. Angew. Chem., Int. Ed. Engl. 1982, 21, 654. (b) See, also: Juaristi, E. Introduction to Stereochemistry and Conformational Analysis, Wiley: New York; 1991, pp 52-54.

12. Dimethyl fumarate was obtained as a white solid, $\mathrm{mp} 105-106{ }^{\circ} \mathrm{C}$ in agreement with literature data $\left(\mathrm{mp} 102-106{ }^{\circ} \mathrm{C}\right.$, see: Aldrich Catalog of Fine Chemicals, Sigma-Aldrich Chemical Company, Milwaukee, 2009-2010; p 1131).

13. Rovnyak, G. C. U. S. Patent 4220 791, 1980.

14. Crystal data for 4: $\mathrm{C}_{18} \mathrm{H}_{21} \mathrm{~N}_{3} \mathrm{O}_{3}$, monoclinic, space group $\mathrm{P} 2(1)$, a = 11.9112(3) $\AA, \mathrm{b}=$ 6.4941(2) $\AA, c=12.0419(4) \AA, \alpha=\gamma=90^{\circ}, \beta=117.880(2)^{\circ}, \mathrm{V}=823.35(4) \AA^{3}$, crystal size: $0.4 \times 0.1 \times 0.075 \mathrm{~mm}^{3}, \mathrm{R} 1=0.0424(w \mathrm{R} 2=0.1028)$. Crystal data for 5: $\mathrm{C}_{11} \mathrm{H}_{12} \mathrm{~N}_{2} \mathrm{O}_{3}$, monoclinic, space group P 2(1)/c, a = 9.1982(2) $\AA, \mathrm{b}=7.2755(2) \AA, \mathrm{c}=16.6637(4) \AA, \alpha=\gamma$ $=90^{\circ}, \beta=100.9830(10)^{\circ}, \mathrm{V}=1094.73(5) \AA^{3}$, crystal size: $0.6 \times 0.27 \times 0.12 \mathrm{~mm}^{3}, \mathrm{R} 1=0.0404$ $(w \mathrm{R} 2=0.0943)$. Crystal data for 11: $\mathrm{C}_{19} \mathrm{H}_{27} \mathrm{~N}_{2} \mathrm{O}_{5}$, orthorhombic, space group P 2(1)2(1)2(1), $\mathrm{a}=6.1885(1) \AA, \mathrm{b}=17.8721(5) \AA, \mathrm{c}=17.8860(4) \AA, \alpha=\beta=\gamma=90^{\circ}, \mathrm{V}=1978.218(77) \AA^{3}$, crystal size: $0.35 \times 0.17 \times 0.12 \mathrm{~mm}^{3}, \mathrm{R} 1=0.0740(w \mathrm{R} 2=0.1516)$. Crystal data for 12: $\mathrm{C}_{16} \mathrm{H}_{15} \mathrm{~N}_{3} \mathrm{O}_{2}$, monoclinic, space group $\mathrm{P} 2(1), \mathrm{a}=8.7768(3) \AA, \mathrm{b}=6.1093(12) \AA, \mathrm{c}=$ 13.596(3) $\AA, \alpha=\gamma=90^{\circ}, \beta=101.76(3)^{\circ}, V=713.7(2) \AA^{3}$, crystal size: $0.5 \times 0.25 \times 0.25 \mathrm{~mm}^{3}$, $\mathrm{R} 1=0.0412(w \mathrm{R} 2=0.1042)$. Crystal data for 15: $\mathrm{C}_{14} \mathrm{H}_{19} \mathrm{~N}_{3} \mathrm{O}_{3}$, orthorhombic, space group $\mathrm{P}$ 2(1)2(1)2(1), $\mathrm{a}=6.4721(2) \AA, \mathrm{b}=10.9192(3) \AA, \mathrm{c}=20.4803(6) \AA, \alpha=\beta=\gamma=90^{\circ}, \mathrm{V}=$ 1447.35(7) $\AA^{3}$, crystal size: $0.27 \times 0.1 \times 0.1 \mathrm{~mm}^{3}, \mathrm{R} 1=0.0471(w \mathrm{R} 2=0.0972)$. CCDC 758525 (for 4), CCDC 758526 (for 5), CCDC 758527 (for 11), CCDC 758528 (for 12) and CCDC 758529 (for 15) contain the supplementary crystallographic data for this paper. These data can be obtained free of charge from The Cambridge Crystallographic Data Center via http://www.ccdc.cam.ac.uk/data_request/cif. 